\title{
CONTROL OF INTERCELLULAR COMMUNICATION BY VOLTAGE DEPENDENCE OF GAP JUNCTIONAL CONDUCTANCE ${ }^{1}$
}

\author{
ANDREW L. HARRIS, ${ }^{2}$ DAVID C. SPRAY, AND MICHAEL V. L. BENNETT \\ Division of Cellular Neurobiology, Rose F. Kennedy Center for Research in Mental Retardation and Human Development, \\ Albert Einstein College of Medicine, Bronx, New York 10461
}

Received April 19, 1982; Revised August 3, 1982; Accepted August 3, 1982

\begin{abstract}
The junctional conductance between coupled amphibian blastomeres exhibits a high degree of voltage dependence, as previously described in voltage clamp studies (Spray, D. C., A. L. Harris, and M. V. L. Bennett (1981) J. Gen. Physiol. 77: 77-95; Harris, A. L., D. C. Spray, and M. V. L. Bennett (1981) J. Gen. Physiol. 77: 95-117). The present study examines the properties which this voltage dependence confers on electrotonic coupling between cells. The effects of applied pulses and ramps of current are studied experimentally and are modeled by calculation. During sufficiently large current pulses applied to one cell of a pair, the cells uncouple and then recouple after termination of the pulses. Ramps of current applied to one of the cells can give voltage-current (V-I) relations with a region of hysteresis within which the cells are stably coupled or stably uncoupled depending on previous history. Intrinsically generated currents are able to cause bistability of coupling in the absence of externally applied current. Calculations from the parameters of junctional conductance defined under voltage clamp fully account for these findings and illustrate how junctional and nonjunctional conductances affect the V-I relations in the region of bistability. Recordings from several cells within a small group show that boundaries of intercellular communication can be altered by applied current, a finding that also can be accounted for by voltage dependence of junctional conductance. The "Appendix" examines quantitatively the criteria required for bistability of coupling and the relevance of bistability for intercellular signaling. The plasticity of coupling which the voltage dependence of junctional conductance confers on cells offers an intriguing mechanism by which patterns of intercellular communication could be determined and changed in developing tissues.
\end{abstract}

Electrotonic coupling is widespread within inexcitable as well as excitable tissues (cf. Bennett and Goodenough, 1978). The coupling pathway for current flow is provided by the intercellular channels which comprise gap junctions. In addition, junctional channels allow intercellular passage of small molecules such as those involved in metabolic cooperation and may play a role in other forms of interaction between cells. Thus, cellular mechanisms which modulate electrotonic coupling may control chemical communication within a tissue, influencing important functions such as tissue growth and differentiation (cf. Bennett et al., 1981a, b; Spray et al., 1982c).

\footnotetext{
${ }^{1}$ We wish to thank Pat Model, David Covell, Bill David, and Steve Leber for the embryonic cells used in this study and Joe Zavilowitz for expert supporting services. A. L. H. was supported by an individual National Research Service Award postdoctoral fellowship. D. C. S. was a McKnight Scholar and is the recipient of a McKnight Foundation Development Award. This work was also supported by National Institutes of Health Grants NS-16524, HD-04248, NS-12627, and NS-07512.

${ }^{2}$ Present address: Department of Biological Sciences, Stanford University, Stanford, CA 94305.
}

We have described and characterized under voltage clamp the voltage dependence of the conductance of gap junctions between amphibian blastomeres (Harris, 1979; Spray et al., 1979, 1981a; Harris et al., 1981). In these cells, junctional conductance is maximal at zero transjunctional voltage and is decreased steeply and symmetrically by transjunctional voltage of either polarity. In the present paper, experiments and mathematical modeling show that voltage dependence of junctional conductance can give rise to regions of negative slope in the voltage-current (V-I) relations of pairs of coupled cells and that current transients (applied or intrinsic) can evoke regenerative and sustained changes in coupling. Furthermore, boundaries of coupling within a population of cells can be altered by changes in intrinsic or applied current.

The possibility of easily produced transitions between stable states of coupling adds substantial flexibility to the potential roles of gap junctions. Under specific and reversible conditions, cells could be coupled uniformly, coupled as small cell groups, or isolated from one another depending on the passive and active properties of the 
junctional and nonjunctional membranes. The plasticity of coupling allowed by voltage dependence of junctional conductance may be important in the determination, differentiation, and development of embryonic tissues in amphibia. Preliminary communications of some of these data have been published (Spray et al., 1978; Bennett et al., 1980; Harris et al., 1982).

\section{Materials and Methods}

Pairs of blastomeres from early cleavage stages (stages 6 to 8; Harrison, 1969) of Ambystoma mexicanum embryos were isolated mechanically and maintained in physiological saline (Flickinger, 1949; Niu-Twitty solution: $58.2 \mathrm{~mm} \mathrm{NaCl}, 0.7 \mathrm{mM} \mathrm{KCl}, 0.3 \mathrm{~mm} \mathrm{Ca}\left(\mathrm{NO}_{3}\right)_{2}, 0.8$

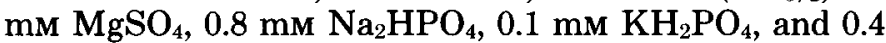
$\mathrm{mM} \mathrm{NaHCO}$, adjusted to $\mathrm{pH} 7.8$ with $\mathrm{NaOH}$ ) as previously described (Spray et al., 1981a). Mitosis was blocked with up to $0.05 \%$ colchicine. Each cell of a pair was impaled with separate voltage and current clectrodes. Rectangular pulses or ramps of current were applied in order to characterize electrotonic coupling. In some experiments, coupling was examined within small clumps of cells isolated from embryos in a similar manner.

The traces of voltage and current were displayed on a Tektronix, Inc. (Beaverton, OR) storage oscilloscope and either photographed with a Grass Instrument Co. (Quincy, MA) camera or recorded on paper by a Gould, Inc. (Cleveland, $\mathrm{OH}$ ) Brush Recorder. Data were digitized by hand with a Hewlett-Packard Co. (Palo Alto, CA) model 9864 digitizer. Computational analysis and modeling were performed either with a Hewlett-Packard model 9830A programmable calculator or a Digital Electronics Co. (Maynard, MA) MINC-11 computer.

\section{Analysis of the Equivalent Circuit}

The equivalent circuit for an isolated pair of coupled blastomeres is shown in Figure $1 A$. The conductances and capacitances of the nonjunctional membranes of each cell can be lumped and assigned individual values. Since the junctional membrane has a very high conductance per unit area, its electrical time constant is small and can be neglected. Analysis of the steady state properties of the circuit is straightforward (Bennett, 1966). Current $(I)$ applied to cell 1 divides between two parallel paths to ground, one through the conductance of the nonjunctional membrane of cell $1\left(g_{1}\right)$ and the other through the series conductances of the junctional membrane $\left(g_{j}\right)$ and the nonjunctional membrane of cell $2\left(g_{2}\right)$. The steady state voltages recorded in cells 1 and 2 in response to current $I$ applied to cell 1 are, respectively:

$$
V_{1}=\frac{g_{2}+g_{j}}{g_{1} g_{2}+g_{1} g_{i}+g_{2} g_{i}} I
$$

and

$$
V_{2}=\frac{g_{j}}{g_{1} g_{2}+g_{1} g_{j}+g_{2} g_{j}} I
$$

The ratio of these voltages is defined as the coupling coefficient $\left(k_{12}\right)$ from cell 1 to cell 2 :

$$
k_{12}=\frac{V_{2}}{V_{1}}=\frac{g_{j}}{g_{2}+g_{j}}
$$

The input conductance of cell $1\left(g_{11}\right)$ may be defined as:

$$
g_{11}=I / V_{1}
$$

Similarly, the transfer conductance from cell 1 to cell 2 $\left(g_{12}\right)$ may be defined as the ratio of the applied current to the voltage in cell 2 :

$$
g_{12}=I / V_{2}
$$

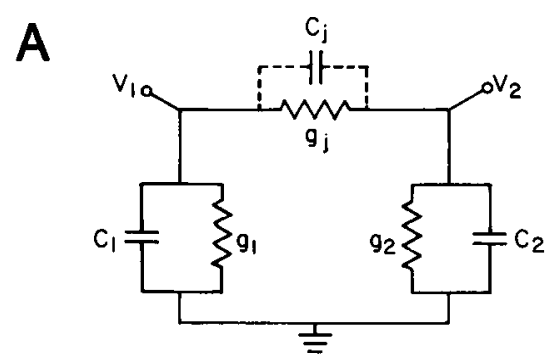

B
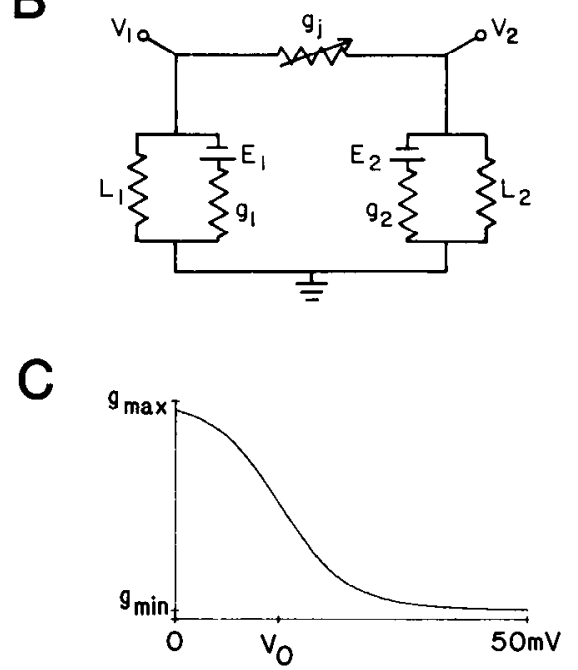

Figure 1. A, Equivalent circuit for an isolated pair of coupled blastomeres. The conductances $g_{1}$ and $g_{2}$ are the nonjunctional conductances of cells 1 and 2, respectively, and $g_{j}$ is the junctional conductance. The electrical capacities of the nonjunctional membranes of the cells are $C_{1}$ and $C_{2}$, respectively. The time constant of the junctional membrane is sufficiently short that its capacity $\left(C_{j}\right)$ may be neglected. The membrane potentials of the two cells are $V_{1}$ and $V_{2} . B$, Equivalent circuit for a pair of coupled blastomeres (capacities neglected) allowing for variation in intrinsic membrane potentials and leakage conductances of the nonjunctional membranes. The intrinsic membrane potentials for cells 1 and 2 are $E_{1}$ and $E_{2}$, respectively. The leakage conductances represented by $L_{1}$ and $L_{2}$ shunt the selectively permeable nonjunctional conductances $g_{1}$ and $g_{2}$. $C$, Steady state conductance-voltage relation for the junctional membrane of amphibian blastomeres (Spray et al., 1981a). The junctional conductance $\left(g_{j}\right)$ falls as a steep function of transjunctional voltage $\left(V_{j}\right)$, and can be well described by a form of the Boltzmann relation (equation 6). At large transjunctional voltages, a minimal conductance $\left(g_{\text {min }}\right)$ is attained which is a few percent of the maximal conductance $\left(g_{\max }\right)$. The junctional conductance is symmetrically sensitive to transjunctional voltages of either polarity. The maximal conductance $\left(g_{\max }\right)$ is not attained at zero transjunctional voltage, due to the sensitivity of the junctional channels to voltages of each polarity, and is estimated by extrapolation. The voltage at which the conductance is half-maximal $\left(V_{0}\right)$ is 14 to $15 \mathrm{mV}$. 
It is sometimes convenient to refer to these parameters as their reciprocals, the input and transfer resistances, respectively:

$$
\begin{aligned}
& r_{11}=1 / g_{11}=V_{1} / I \\
& r_{12}=1 / g_{12}=V_{2} / I
\end{aligned}
$$

\section{Voltage dependence of junctional conductance}

The previous voltage clamp studies showed that $g_{j}$ could be described as a simple function of transjunctional voltage $\left(V_{j}\right)$ (Fig. $1 C$; Spray et al., 1981a; Harris et al., 1981). For steady state conditions, its value $g_{\infty}$ was well fit by the Boltzmann equation of the form:

$$
g_{\infty}=\frac{g_{\max }-g_{\min }}{1+\exp \left[A\left(V_{j}-V_{0}\right)\right]}+g_{\min }
$$

where $g_{\max }$ is the maximum value of $g_{j}, g_{\min }$ is the minimum value of $g_{j}$ that can be obtained with large transjunctional voltages, $A$ is a constant expressing voltage sensitivity, and $V_{0}$ is the voltage at which $g_{\infty}$ is halfway between $g_{\max }$ and $g_{\min }$. The parameters of voltage sensitivity used in this paper were those determined in the previous voltage clamp studies (Spray et al., 1981a; Harris et al., 1981): $g_{\min }=0.04 g_{\max }, A=0.217$, and $V_{0}$ $=14.7 \mathrm{mV}$. For relaxations at constant transjunctional voltage following a step from a transjunctional voltage of the same polarity or from zero, $g_{j}$ followed an exponential of the form:

$$
g_{j}=g_{\infty}+\left(g_{0}-g_{\infty}\right) \exp (-t / \tau)
$$

where $g_{0}$ is the initial value of $g_{j}, g_{\infty}$ is the steady state value of $g_{j}$ for the new voltage $V_{j}, t$ is time and $\tau$ is the time constant of relaxation. The measured parameters $g_{\infty}$ and $\tau$ were used to calculate opening and closing rates according to $\alpha=G_{\infty} / \tau$ and $\beta=\left(1-G_{\infty}\right) / \tau$, where $G_{\infty}$ is the normalized steady state conductance given by $G_{\infty}=$ $\left(g_{\infty}-g_{\min }\right) /\left(g_{\max }-g_{\min }\right)$. These rates were well fit by exponential functions of $V_{j}$ :

$$
\begin{gathered}
\alpha=\lambda \exp \left[-A_{\alpha}\left(V_{j}-V_{0}\right)\right] \\
\beta=\lambda \exp \left[A_{\beta}\left(V_{j}-V_{0}\right)\right]
\end{gathered}
$$

where $\lambda=0.0013, A_{\alpha}=0.077$, and $A_{\beta}=0.14$ (Harris et al., 1981). For reasons detailed under "Results," instead of allowing the closing rate to increase indefinitely with large $V_{j}, \beta$ was made to asymptotically approach a limit of $20 / \mathrm{sec}$ (minimum $\tau$ of $50 \mathrm{msec}$ ) according to the relation:

$$
\beta^{\prime}=\beta /(1+50 \beta)
$$

This equation satisfactorily fit the voltage clamp data over the range of measurement. To incorporate this adjustment in the calculations, $g_{\infty}$ at a given voltage was calculated according to:

$$
g_{\infty}=\left(g_{\max }-g_{\min }\right) \frac{\alpha}{\left(\alpha+\beta^{\prime}\right)}+g_{\min }
$$

where $\alpha$ is evaluated according to equation 7 and $\beta^{\prime}$ is evaluated according to equations 8 and $8 \mathrm{a}$.

\section{Transient effects of applied current}

For computation of the transient effects of current pulses (Fig. 3), the voltage in each cell was calculated iteratively. For each time increment $\Delta t$, the change in voltage in each cell, $\Delta V_{1}$ or $\Delta V_{2}$, was the product of the current charging the capacity $\left(C_{1}\right.$ or $\left.C_{2}\right)$ of that cell and the time increment. Thus:

$$
\begin{gathered}
\Delta V_{1}=\frac{\left(I-g_{1} V_{1}-I_{j}\right) \Delta t}{C_{1}} \\
\Delta V_{2}=\frac{\left(I_{j}-g_{2} V_{2}\right) \Delta t}{C_{2}}
\end{gathered}
$$

where $I_{j}$ is the junctional current, given by $I_{j}=g_{j}\left(V_{1}-\right.$ $V_{2}$ ) where $g_{j}$ is conductance calculated iteratively according to:

$$
\Delta g_{j}=\left[\left(g_{\max }-g_{j}\right) \alpha-\left(g_{j}-g_{\min }\right) \beta^{\prime}\right] \Delta t
$$

For matching the experimental data, we chose $g_{1}, g_{2}$, and $g_{\max }$ to fit steady state values obtained at small transjunctional voltages, and we chose $C_{1}=C_{2}=15 n F$, assuming cell size constant, to give a time constant of 15 msec for a $1.0 \mu$ mho conductance cell. For calculation of the effects of current pulses, $I$ was incremented appropriately for the calculations for each time increment. In all cases, the time increments used were such that the use of smaller increments did not alter the result of the calculations.

For calculation of the effects of ramps of current (Fig. 5 ), the time constants of the nonjunctional membranes could be ignored due to the slow rate of change of applied current. The only time-variant parameter was the junctional conductance, evaluated for each time increment according to equation 12 , and the voltages were calculated according to equations 1 and 2. Representative calculations including the nonjunctional time constants (equations 10 and 11) yielded essentially identical results.

\section{Steady state V-I relations}

The steady state behavior of pairs of cells was calculated with the independent variable being either applied current or transjunctional voltage. The former method mimics the application of a ramp of current to measure the steady state V-I relations of coupled cells and results in discontinuities at the thresholds for uncoupling and recoupling. The latter method yields continuous V-I relations which are three-valued in current between the thresholds for uncoupling and recoupling, the middle values being inaccessible in current clamp experiments. Aside from this middle region, the V-I relations of the cells from the two methods are identical, while the method of varying transjunctional voltage has the advantage of requiring much less computing time.

Method of varying current. With initial values being $V_{1}=V_{2}=V_{j}=0$ and $I=0$, current was successively incremented by a small value $\Delta I$. When $I$ became sufficiently large, the sign of $\Delta I$ was changed and the process continued until $I$ was again zero. After each increment in current, $V_{1}$ and $V_{2}$ were calculated from equations 1 and 2 and $g_{j}$ equal to its steady state value $g_{\infty}$ was calculated from equations 7 to 9 , where $V_{j}=V_{1}-V_{2}$. The values of interest were plotted connected by lines to the previous values, and current incremented if the change in $g_{j}$ was below a criterion level of $0.01 \%$. Otherwise, the calculation of voltages and $g_{j}$ was repeated with their new values without increment in $I$ until the change between the last 
two values of $g_{j}$ was less than the criterion level. This procedure preserved the accuracy of calculation near threshold for transitions between coupled and uncoupled states and also produced the vertical transitions at currents incrementally above the thresholds. The increment of current was decreased until the calculated responses were of essentially constant form.

Method of varying transjunctional voltage. For each value of $V_{j}$, steady state values of $g_{j}=g_{\infty}$ were calculated according to equations 7 to 9 . Since $I_{j}=g_{j} V_{j}, V_{2}=I_{j} / g_{2}$, $V_{1}=V_{2}+V_{j}$, and applied current $I=g_{1} V_{1}+I_{j}$, the parameters $I, V_{1}$, and $V_{2}$ can be obtained as functions of $V_{j}$ :

$$
\begin{gathered}
V_{1}=V_{j}\left(1+g_{j} / g_{2}\right) \\
V_{2}=V_{j} g_{j} / g_{2} \\
I=V_{1} g_{1}+V_{j} g_{j}
\end{gathered}
$$

The voltages were calculated for ranges of $V_{j}$ which covered the desired ranges of applied current with small enough differences between successive values of $V_{j}$ to yield smooth curves.

To give steady state $\mathrm{V}$-I relations like those obtained by varying current, the middle region of the continuous V-I relations was deleted and vertical lines were drawn in at the thresholds as indicated in the inset of Figure 5 (see also Figs. 8 and 9).

In some cases (Fig. 8), it was of interest to calculate the $\mathrm{V}$-I relations for cells could have unequal resting potentials $\left(E_{1}, E_{2}\right)$ to which could be added leakage conductances $\left(L_{1}, L_{2}\right)$ to zero voltage through their nonjunctional membranes (equivalent circuit shown in Fig. $1 B$ ). Although the figures showing these calculations are discontinuous, they were calculated in terms of $V_{j}$ according to equations generalized from equations 13 to 15 :

$$
\begin{gathered}
V_{1}=V_{j}+\frac{V_{j} g_{j}+E_{2} g_{2}}{g_{2}+L_{2}} \\
V_{2}=\frac{V_{j} g_{j}+E_{2} g_{2}}{g_{2}+L_{2}} \\
I=V_{j} g_{j}+V_{1}\left(g_{1}+L_{1}\right)-E_{1} g_{1}
\end{gathered}
$$

The V-I relations for three linearly arranged cells (Fig. 12) were calculated by the method of varying current and the equations:

$$
\begin{gathered}
V_{1}=\frac{I}{g_{1}+g_{2}^{\prime} g_{j 1} /\left(g_{2}{ }^{\prime}+g_{j 1}\right)} \\
V_{2}=V_{1} g_{j 1} /\left(g_{2}{ }^{\prime}+g_{j 1}\right) \\
V_{2}=V_{2} g_{j 2} /\left(g_{3}+g_{j 2}\right)
\end{gathered}
$$

where $g_{2}{ }^{\prime}=g_{2}+g_{j 2} g_{3} /\left(g_{j 2}+g_{3}\right), g_{3}$ is the nonjunctional conductance of cell 3 , and $g_{j 1}$ and $g_{j 2}$ are the conductances of the junctions between cells 1 and 2 and between cells 2 and 3 , respectively. For these calculations, $g_{j 1}$ and $g_{j 2}$ were evaluated using equations 7 to 9 where the relevant transjunctional voltages were $V_{1}-V_{2}$ and $V_{2}-V_{3}$, respectively.

\section{Results}

Effect of current pulses on coupling. A small constant current pulse applied to one blastomere of a coupled pair produced constant voltages in both cells once the membrane capacity had been charged (Fig. $2 A_{1}$ ). During larger current pulses, the potential in the directly polarized cell increased and electrotonic spread of potential to the other cell decreased in a sigmoidal fashion (Fig. 2, $A_{2}$ to $A_{4}$ ). The uncoupling occurred over periods of several tenths of seconds to several seconds, beginning at shorter latencies and proceeding more rapidly with larger polarizations. Similar changes in coupling occurred during current pulses applied in the other cell (Fig. 2B) and when the polarity of the applied current was reversed (Fig. 2C). The symmetry of responses follows from the electrical linearity of the nonjunctional membrane of these cells and symmetry of the voltage-dependent changes in junctional conductance around zero transjunctional voltage (Spray et al., 1981a). During a constant current pulse, the coupling coefficient (equation 3) for cell pairs could decay from 0.8 to less than 0.05 . Cells remained stably uncoupled during long current pulses (100 sec was the longest duration tested).

The uncoupling observed during applied current pulses appears to be regenerative, and the voltages in the cell to
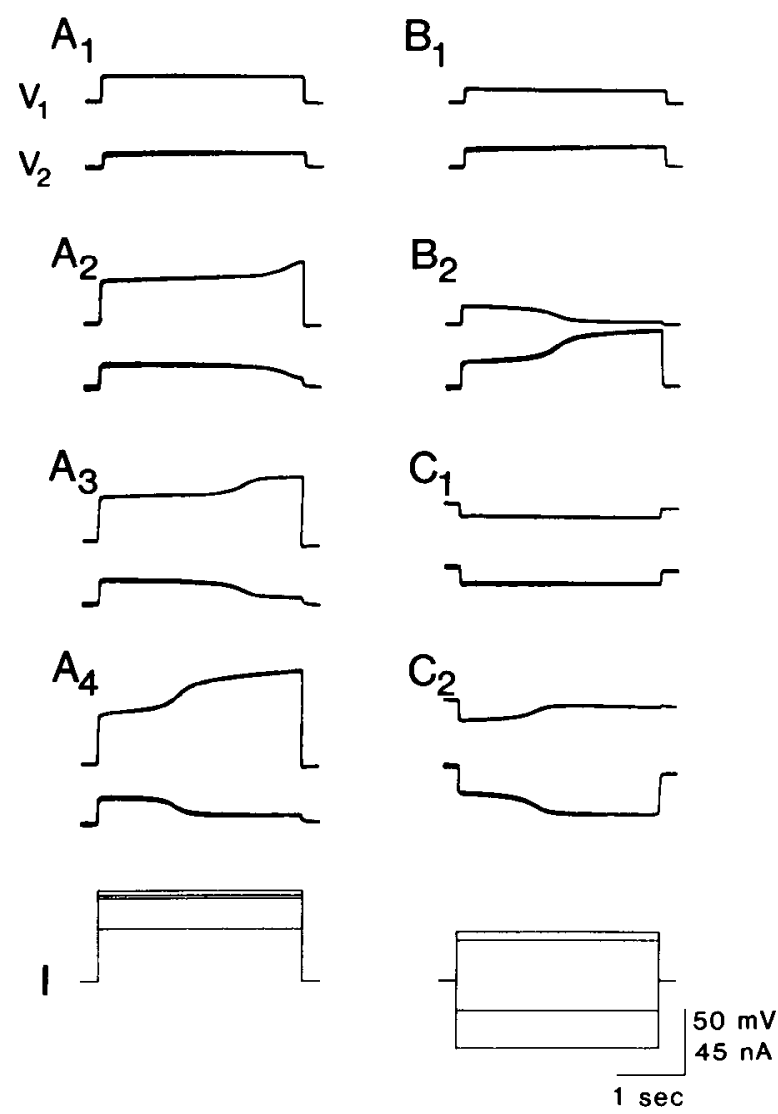

Figure 2. Effects of applied current on coupling in an isolated pair of cells. $A$, Current applied to cell 1 . For the smallest current $(\mathrm{I})$, the potentials in cell $1\left(V_{1}\right)$ and cell $2\left(V_{2}\right)$ remained nearly constant. Near the end of a larger current pulse $\left(A_{2}\right)$ the input resistance of cell 1 increased and the electrotonic coupling to cell 2 decreased. With still larger currents $\left(A_{3-4}\right)$, the uncoupling occurred earlier. $B$ and $C$, Similar effects were seen when depolarizing $(B)$ or hyperpolarizing $(C)$ currents were applied to cell 2. Currents $(I)$ corresponding to each column are shown at the bottom. 
A
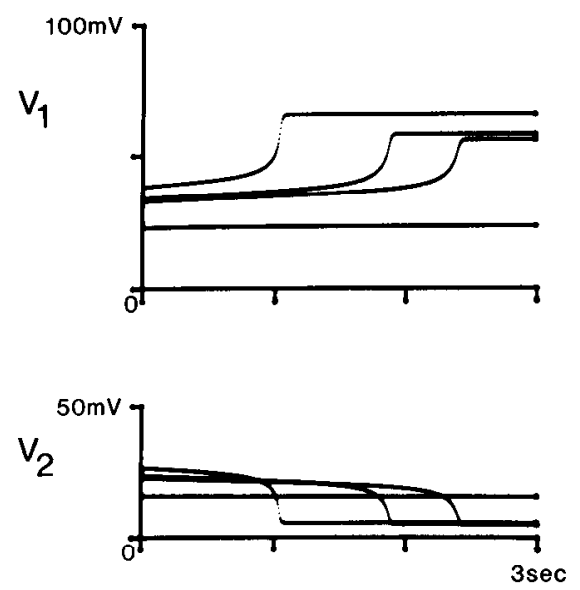

B
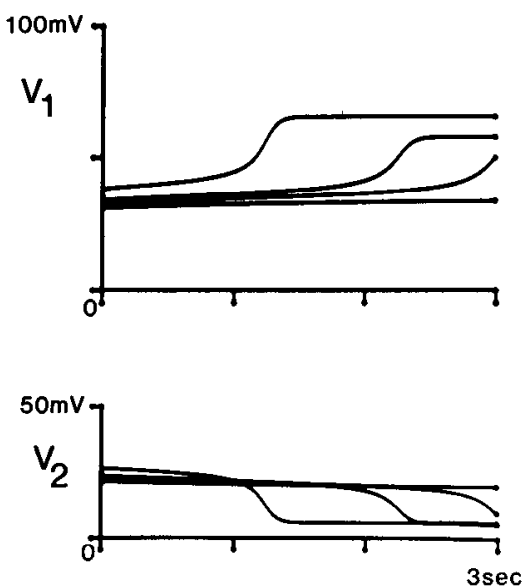

Figure 3. Calculated effects of current pulses on a pair of coupled cells where junctional conductance is voltage dependent (equations 10 to 12). The voltages in the two cells were calculated for the currents applied in Figure 2 and values of conductances were chosen to approximate the coupling observed for small currents: $I=37,53,55$, and $62 \mathrm{nA} ; g_{1}=0.85 \mu \mathrm{mho} ; g_{2}=1.1 \mu \mathrm{mho}$; and $g_{m a x}=2.6 \mu \mathrm{mho}$. $A$, Results of calculations without limiting the closing rate constant. The rate constant $(\beta)$ governing the voltage-dependent closing of the junctional channel was allowed to increase without limit according to equation 8 . For the larger currents, the calculated final approach of $V_{1}$ and $V_{2}$ to the steady state levels occurs much more rapidly than in the experimental data of Figure 2. $B$, Results of calculations with limitation of rate constant. The rate constant $\beta$ was limited to a maximum of 20 / sec according to equations 8 and 8 a (minimum time constant of $50 \mathrm{msec}$ ). With this limitation, the calculated time course of uncoupling during applied current pulses closely approximates the experimental data of Figure 2.

which current pulses were applied resemble prolonged action potentials (Fig. 2). As discussed quantitatively in the "Appendix," the uncoupling is regenerative because of voltage dependence of junctional conductance. When current applied in one of the cells is sufficient to produce a transjunctional voltage at which junctional conductance decreases, the input conductance of the cell decreases and the applied current then produces a larger transjunctional voltage. The larger voltage causes a further decrease in junctional conductance and the changes continue in a classically regenerative manner.

The conductance of the second cell also affects the regenerative behavior (see "Appendix"). The fraction of the voltage in the first cell which develops across the junctions depends on the conductances of the junctional membrane and the nonjunctional membrane of the second cell (equation 3). With a higher conductance second cell, there is a smaller fractional change in transjunctional voltage for a given change in junctional conductance. When the second cell is of lower conductance, a given change in junctional conductance will cause a larger change in transjunctional voltage, and the process is more regenerative.

Calculations of uncoupling during current pulses. The effects of current pulses on a pair of coupled cells were calculated (equations 10 to 12) for the case where nonjunctional conductance is linear and junctional conductance is voltage dependent with parameters as reported in our previous voltage clamp studies (Spray et al., 1981a; Harris et al., 1981). Comparison of the current clamp data to these calculations provides a test of the adequacy of our description of the voltage dependence and of whether it fully accounts for the behavior of the cells under current clamp.
Figure $3 A$ shows the calculated voltages of a pair of cells for current pulses of several magnitudes chosen to correspond to the currents applied in Figure 2A. The values of $g_{1}, g_{2}$, and $g_{\max }$ were chosen to approximate the observed coupling for small currents in Figure 2. There is good agreement between these calculations and the data of Figure 2 except for two minor discrepancies.

For the larger currents, the calculated final approach to the steady state levels occurs much more rapidly than in the experimental data. The transjunctional voltages during this phase of uncoupling under current clamp were 15 to $25 \mathrm{mV}$ larger than those imposed in the voltage clamp experiments in which the rate constants for junctional conductance change were determined. The calculations in Figure $3 A$ used rates defined by extrapolations of equations 7 and 8 well beyond the range of measurement. It is reasonable that the closing rate saturates at large transjunctional voltages instead of continuing to increase exponentially (and such a trend may occur in the voltage clamp data; Harris et al., 1981). Therefore, for the calculations shown in Figure $3 B$, the rate constant of closing was made to asymptotically approach a limiting value as described by equation $8 \mathrm{a}$. Although we did not systematically investigate the effect of varying the limiting rate, a value of $20 / \mathrm{sec}$ (corresponding to a minimum time constant of $50 \mathrm{msec}$ ) gave results closely approximating the current clamp data. The few voltage clamp experiments where large transjunctional voltage steps were imposed also suggested a maximum closing rate of about this value (unpublished observation).

The second discrepancy is that, following uncoupling by the larger depolarizing currents, the experimental data show an upward drift of the plateau voltage of the cell to 
which the currents were applied (Fig. $2, A_{3}, A_{4}$, and $B_{2}$ ), while the calculated plateau voltages are constant. Presumably, this upward drift was due to decreasing conductance of the nonjunctional membrane during the current pulse. Voltage clamp studies on single blastomeres (Spray et al., 1981a) showed linearity of nonjunctional conductance over a slightly smaller range of voltages. Recently, we have found that the nonjunctional membrane becomes nonlinear at the larger voltages (unpublished observation), whereas in the calculations, the nonjunctional conductances were assumed to be constant.

Effects of slow current ramps on coupling. The records of Figure 2 demonstrate that coupling is a function of applied current. In order to obtain quasi-steady state VI relations, ramps of slowly increasing and decreasing current were applied to one cell of a pair and the resulting V-I relations were measured as illustrated in Figure 4. During increasing current, the cells were well coupled until the applied current reached approximately $130 \mathrm{nA}$. As current increased a few nanoamperes further, $V_{1}$ rapidly increased and $V_{2}$ decreased; the cells became weakly coupled. With continued increasing current, the

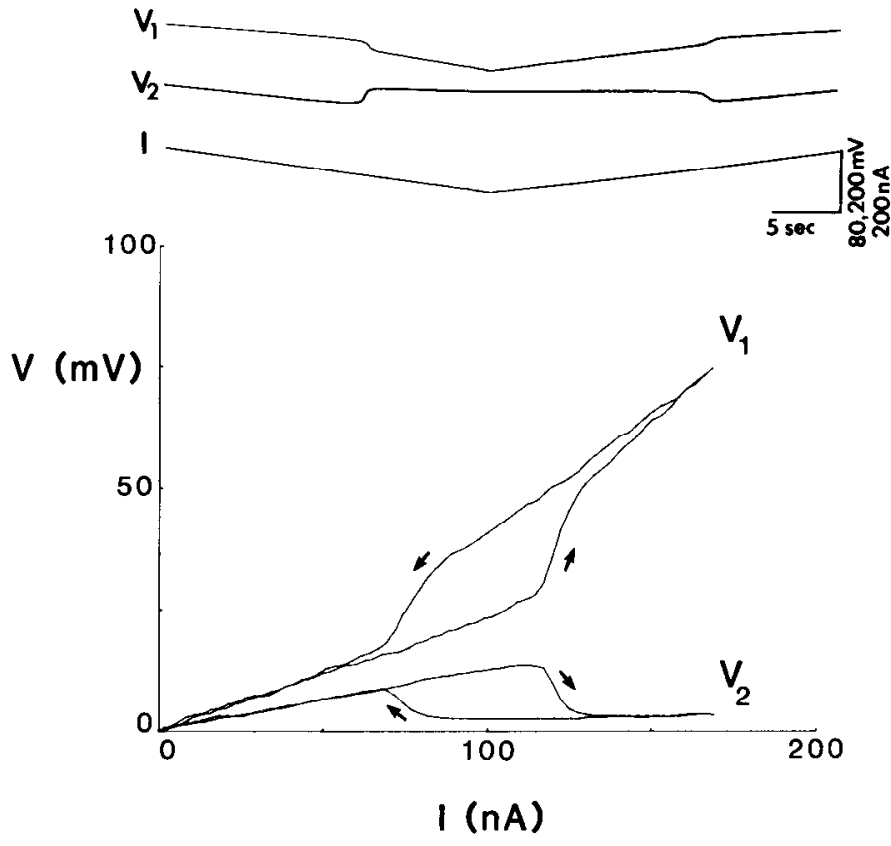

Figure 4. Effects of slow ramps of applied current on coupling. A slowly rising and falling ramp of current $(\sim 30 \mathrm{sec}$ in each direction) was applied to cell 1 in order to obtain a quasisteady state V-I relation. In the upper portion of the figure are the applied current $(I)$ and the resulting voltages in the two cells $\left(V_{1}\right.$ and $\left.V_{2}\right)$. In the lower portion of the figure, $V_{1}$ and $V_{2}$ are plotted as a function of $I$. As the current increased, at a current level of about $130 \mathrm{nA}$, the cells largely uncoupled and remained so as the ramp continued upward. As the ramp of current decreased from its peak, the cells remained coupled until the current reached a level lower than that at which the cells had uncoupled on the upward leg of the ramp. The observed hysteresis and bistability in the V-I relation follows from the voltage dependence of the junctional conductance and reflects the regenerative nature of the uncoupling and recoupling events. The V-I relation was processed by an exponential smoothing algorithm to eliminate noise introduced by digitization error. cells remained weakly coupled. As current decreased from its peak, the cells remained weakly coupled until the applied current was approximately $85 \mathrm{nA}$, at which point $V_{1}$ rapidly decreased and $V_{2}$ increased; the cells recoupled to nearly their initial level. With continued decreasing current, the cells remained well coupled. Thus, in the intermediate range of 85 to $130 \mathrm{nA}$, the cells were in either uncoupled or coupled states depending on whether the applied current approached this range from higher or lower levels. The V-I relations and coupling thus show hysteresis for rising and falling current ramps.

The hysteresis can be explained in terms of voltage dependence of the junctional conductance. When a current adequate to produce regenerative uncoupling is reached, the cells uncouple and a much higher transjunctional voltage is reached. Because the input conductance of the polarized cell is now lower than when the cells were coupled, a smaller applied current can produce a large enough transjunctional voltage to keep the junctional conductance low. Applied current must be reduced substantially below the level which caused uncoupling in order for the cells to recouple. As described below, recoupling is also regenerative.

Calculations of effects of slow current ramps. The effects of ramps of current applied to a pair of coupled cells were calculated for various rates of change of applied current (Fig. 5, curvilinear transitions). The voltages of the two cells were calculated according to equations 1 and 2 for values of $g_{1}, g_{2}$, and $g_{\max }$ which approximated the coupling shown in Figure 4 . In general form, the calculations reproduce the data of Figure 4. With increasing current, the slopes of the relations (the input and transfer resistances) are constant until the cells uncouple, after which they are constant at new values. With decreasing current, the calculations show hysteresis similar to that seen in Figure 4; the cells recouple at lower current levels than those which caused them to uncouple. However, the transitions from coupled to uncoupled states and back occur over a narrower range and at lower and higher levels of current, respectively, when the rate of change of current is slower. The limiting case of the steady state is treated below. Once the transitions are complete and the V-I relations are linear, the cells are coupled or uncoupled, and the input and transfer resistances are independent of the rate of rise or fall of the current ramp. These calculations included a limit on the closing rate (equation $8 \mathrm{a}$ ) as in the calculations for rectangular current pulses, since allowing the closing rate to increase exponentially without limit led to transitions whose terminal phases were too rapid.

There is a minor difference between the calculations shown in Figure 5 and the data in Figure 4. The calculated relation between $V_{1}$ and current in the uncoupled state is the same for increasing and decreasing current and extrapolates to intersect the origin, while in the experimental data the slopes differ slightly for the two directions of current change and extrapolate to intersect the current axis to the right of the origin. A decrease in nonjunctional conductance at large voltages would account for this difference, as it does for the upward drift of the plateau voltage during large rectangular current pulses in Figure 2. 


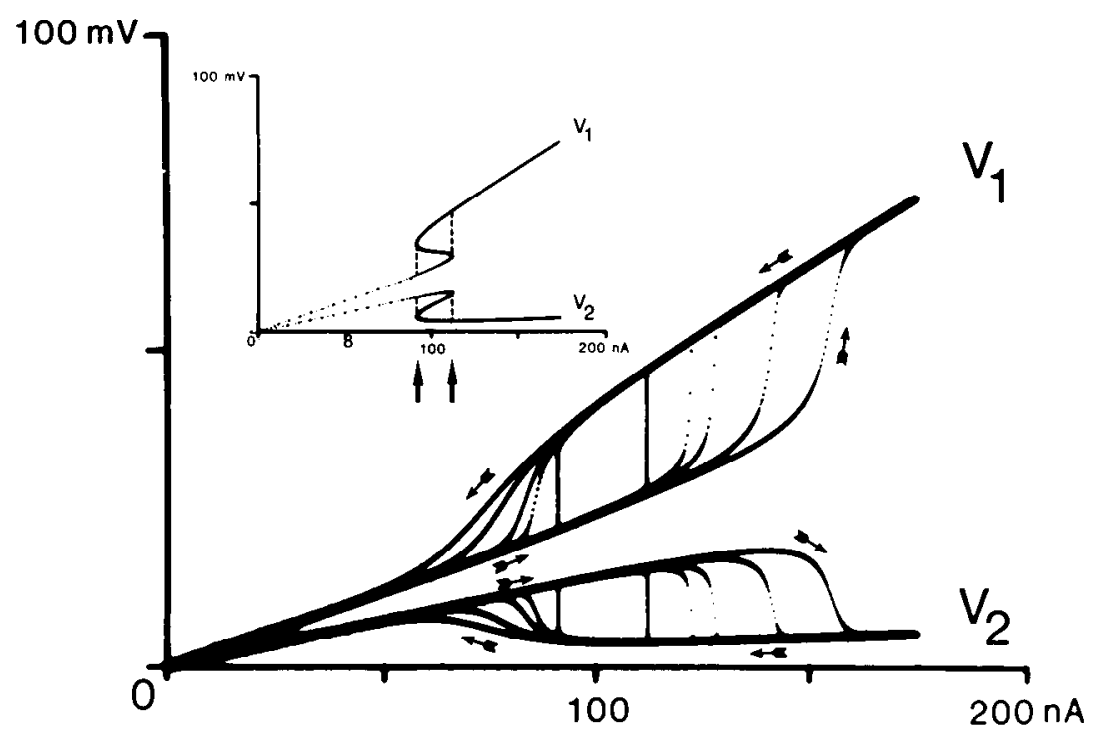

Figure 5. Calculated V-I relations due to applied current ramps and in steady state. The effects of ramps of current applied to a pair of coupled cells were calculated for various rates of change of applied current (curvilinear transitions). The voltages of the two cells were calculated according to equations 1 and 2 for values of conductance chosen to fit the observed coupling in Figure 4 for small currents; $g_{1}=2.1, g_{2}=3.5$, and $g_{\max }=6.5 \mu \mathrm{mho}$. The calculations show hysteresis similar to that seen in the data of Figure 4. When the rate of change of current is slower, the region of current where the cells are either coupled or uncoupled (the region of bistability) is narrower, and the transitions to uncoupled and recoupled states occur at higher and lower levels of current, respectively. The steady state V-I relations (vertical transitions) were calculated for the same values of the conductances (see explanation of $i$ set). The steady state relation is converged upon by the V-I relations during increasingly slow ramps of current (calculations are shown for pairs of rising and falling ramps where the duration of each ramp was 10, 30, 60, and 120 sec). The voltages in the cells are discontinuous functions of applied current where the steady state V-I relations are perpendicular to the current axis. The points where the transitions begin represent rheobasic thresholds for uncoupling and recoupling as current rises and falls. Inset, Continuous V-I relations (solid lines) were calculated for the above parameters by letting transjunctional voltage be the independent variable and calculating the necessary applied current and the voltages in each cell (equations 13 to 15 ). The vertical dashed lines indicate where the steady state relations calculated by varying current diverge from the continuous relations at the rheobasic thresholds for uncoupling and recoupling; these lines correspond to the vertical transitions of the larger graph.

Calculation of steady state and continuous V-I relations. The steady state V-I relations were calculated (see "Materials and Methods") for the same values of the conductances as for the V-I relations of current ramps and also plotted in Figure 5 . The steady state voltages in the cells are discontinuous functions of applied current where the voltage-current relations are perpendicular to the current axis (vertical lines in Fig. 5). These relations are converged upon by the voltage-current relations of increasingly slow ramps of current; during the ramps, the applied current changes too rapidly for the junctional conductance to be at steady state. The points in the steady state relations where the transitions begin represent rheobasic thresholds for uncoupling and recoupling as current rises and falls. The continuous V-I relations in the inset of Figure 5 were those used to obtain the steady state relations in the lower part of the figure.

In the region of hysteresis in the steady state V-I relations, the continuous V-I relations are three-valued for each current (between arrows on current axis). The intermediate values lie on the portion of the curve connecting the threshold points of the steady state relation. In this region, the slope of the relation between current and transjunctional voltage is negative, as may be seen from the larger graph by subtracting $V_{2}$ from $V_{1}$; the slope of $V_{2}$ as a function of $I$ is greater. In this intermediate segment of the continuous V-I relation, the system is in unstable equilibrium. If the two cells were voltage clamped to the intermediate voltages corresponding to a current $I$ in the region of bistability, the clamping currents in cells 1 and 2 would relax to $I$ and zero, respectively; if the voltage control were removed while the currents were maintained at $I$ and zero, the cells would eventually shift to either the coupled or uncoupled state.

Regenerative uncoupling, thresholds, and bistability. The presence of a negative slope region in the V-I relations implies that the uncoupling and recoupling can occur regeneratively and have thresholds. The rheobasic thresholds of uncoupling and recoupling occur at those levels of current where the slopes of the continuous V-I relations become infinite. At rheobasic threshold for uncoupling, a small increase in transjunctional voltage causes $g_{j}$ to decrease, further increasing transjunctional voltage and leading to a further decrease in $g_{j}$, and so on. At rheobasic threshold for recoupling, a small decrease in transjunctional voltage causes $g_{j}$ to decrease, further decreasing transjunctional voltage, leading to a further decrease in $g_{j}$. The regenerative nature of the uncoupling and recoupling events produces the abrupt transitions between states seen in the steady state voltage-current relations in Figure 5.

For constant currents in the range where the continuous V-I relation is three-valued, the system is bistable. If the current is stepped to such a level from zero, the cells 
remain coupled. A brief pulse exceeding the rheobasic threshold can uncouple the cells and they will then remain uncoupled as long as the initial current is applied. A brief pulse in the opposite direction (below the rheobasic threshold for recoupling) can then recouple the cells.

The process of pulse-mediated transitions between stable states of coupling is illustrated in Figure 6. When superimposed on a subthreshold current (solid lines), a small triggering pulse can cause the cells to uncouple (short-dashed lines) or not (long-dashed lines) depending on a small difference in its duration. An oppositely directed pulse can restore coupling. The data illustrate the bistability of coupling and that uncoupling exhibits a threshold. With this type of experiment, one could measure thresholds as the voltages at the end of brief pulses lriggering the transition between coupled and uncoupled states, from which the system relaxed to either state. The values at these voltages would depend on the level of the maintained current. They would also depend on the duration of the triggering pulse because of membrane time constants and time constants of the voltagedependent changes in junctional conductance. The points of unstable equilibrium in the continuous $\mathrm{V}$-I relations would only approximate these thresholds because the pulses would not reach steady state values of current and voltage (see Bennett et al., 1970).

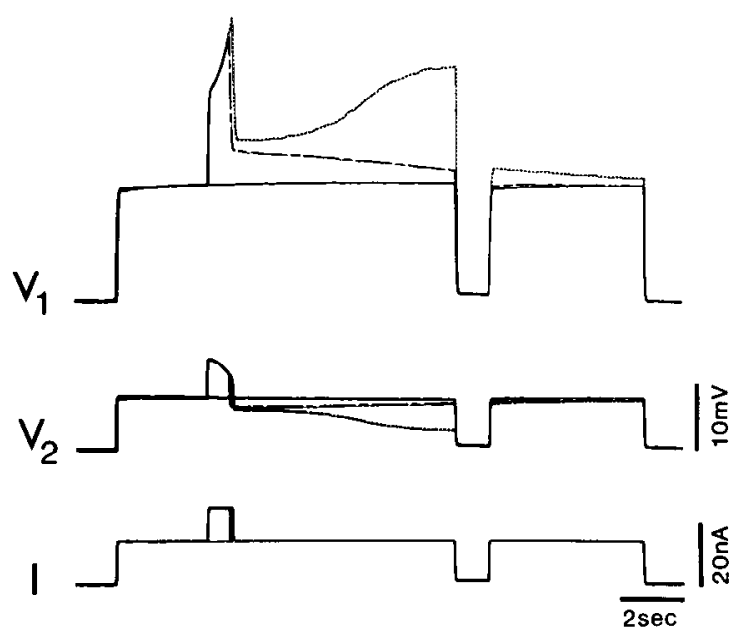

Figure 6. Pulse-mediated transitions between stable states of coupling (three superimposed traces). A long-lasting current pulse $(I)$ was given to cell $1\left(V_{1}\right)$ somewhat below the level required to uncouple it from cell $2\left(V_{2}\right)$ (solid lines). During a short current pulse superimposed on the longer pulse, the input resistance of cell 1 and the transjunctional voltage increased (dashed lines). The brief pulse brought the junctions close to threshold for uncoupling, and with small variations in its duration, it was followed either by uncoupling (short-dashed lines) or by a return to the initial level of coupling (long-dashed lines); that is, either $V_{1}$ increased to a high plateau and $V_{2}$ fell to a low level or the voltages of both cells returned toward their original levels. At a later time, the longer pulse was terminated for a brief interval. When the current was reapplied it was seen that when the cells had been uncoupled (short dashed lines), they largely recoupled during this interval and continued to recouple during the remainder of the pulse. This experiment illustrates the bistability of coupling and a threshold for uncoupling.
Bistability in the absence of applied current. The existence of regions of bistability in the V-I relations depends on the nonjunctional conductances and the junctional conductance and its voltage sensitivity (see "Appendix"). The position of the relations on the V-I plane, and thus the sensitivity of the system to applied or transient intrinsic currents, is a function of the intrinsic resting potentials of the two cells. In principle, currents due to differences in these potentials could bring the cells into the region of bistability so that the cells would be stable in either state of coupling in the absence of applied current (see below).

Bistability without externally applied current occasionally was observed and was ascribable to a difference in resting potentials of the two cells due to leaks produced during penetration of the cells by the microelectrodes (Fig. 7). In the experiment of Figure 7, small hyperpolarizing current pulses of increasing amplitude were delivered to cell 1. Decreases in junctional conductance produced by the current pulses summated to uncouple the cells (Fig. 7, $A$ and $B$, first arrows) and their resting potentials changed in opposite directions. Small depolarizing pulses restored coupling (second arrows). Thus, when coupled cells have sufficiently different resting potentials, current pulses can cause transitions between stable states of coupling and uncoupling which are associated with stable changes in membrane potential.

This experiment demonstrates the bistability without externally applied current which voltage-dependent junctions can confer upon coupling between cells with different resting potentials. It also demonstrates summation of the effects of successive current pulses to cause uncoupling (Fig. 7, $A$ and $B$, before first arrows) and recoupling (Fig. $7 A$, following second arrow) where a single pulse is insufficient. The changes produced by successive pulses summate because there is insufficient time between pulses for full recovery of junctional conductance.

At termination of a pulse uncoupling a pair of cells, their membrane potentials return toward their individual resting potentials (Fig. 7, $A$ and $B$ ), which may be different. The extent to which they approach their resting potentials depends upon the difference between the potentials and the degree to which the cells are uncoupled by the current pulse. If the difference in membrane potentials is sufficient, the cells become stably uncoupled as seen in Figure $7, A$ and $B$ (between arrows). If the difference is marginal, the membrane potentials may require considerable time to converge toward their original levels (Fig. 7C). The transient offsets following uncoupling may appear to be active processes because they outlast the stimulus but in fact are the result of passive (though regenerative) changes in junctional conductance. The transient offsets are necessarily of opposite sign in the two cells.

Calculation of steady state $V \cdot I$ relations for cells with intrinsically generated currents. The conditions under which a leakage conductance provides the current to cause bistability without externally applied current were modeled using equations 16 to 18 , and the results are illustrated in Figure 8 . The relations were calculated for various values of leakage conductance in each cell. The upper graphs show the V-I relations without leakage 

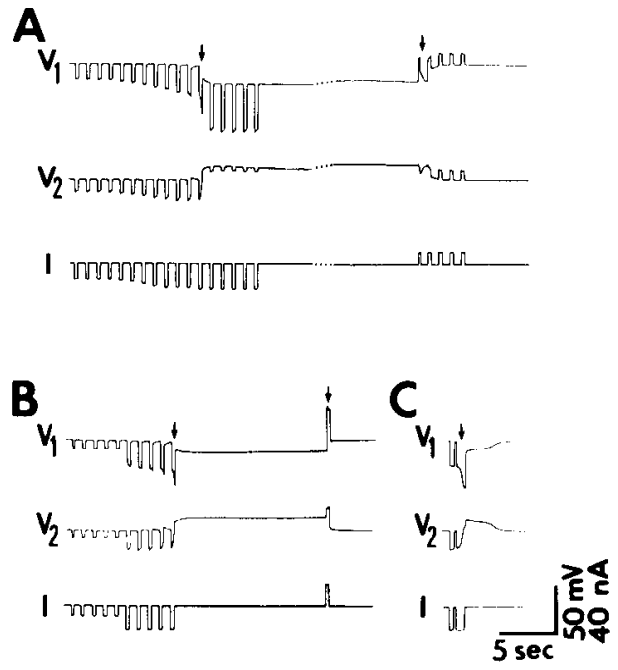

Figure 7. Bistability in the absence of applied current. $A$, Small hyperpolarizing current pulses $(I)$ of increasing amplitude were delivered to cell $1\left(V_{1}\right)$; the smaller pulses show that it was well coupled to cell $2\left(V_{2}\right)$. Partial uncoupling occurred during the pulses as they increased in amplitude. At the termination of one pulse ( first arrow) there was a sudden transition where the cells assumed new resting potentials, cell 1 hyperpolarized and cell 2 depolarized from their previous resting potentials. At this point they were uncoupled (in the absence of externally applied constant current), as indicated by the stable difference in resting potentials and by the decreased coupling during the current pulses following the first arrow. At the second arrow, depolarizing current pulses were applied to cell 1 . The first pulse increased junctional conductance to some extent, but the cells largely uncoupled following the pulse. The second pulse initiated recoupling which persisted as shown by the recovery of coupling indicated by the brief pulses and by restoration of the original membrane potentials. The dotted line indicates a gap of $100 \mathrm{sec}$. $B$, A similar sequence of uncoupling and recoupling. At the second arrow, a single pulse increased the junctional conductance and recoupled the cells as shown by the changed resting potentials. $C$, Transient uncoupling in the absence of applied current. In the same cells as in $A$ and $B$ above, but at a later time, a single pulse did not uncouple the cells. The second, longer pulse caused uncoupling as evidenced by the small transient in $V_{2}$ at the end of the pulse and by the change in membrane potentials of the cells. The uncoupling persisted briefly, and in the absence of further applied current, the cells spontaneously recoupled, recovering their original resting potentials.

conductance in either cell. A leak in cell 1 shifts the V-I relations to the left and enlarges the current range of bistability (right column). If the leak is sufficiently large ( $L=4$ ), the range of bistability includes zero current, and the cell pair can be either stably coupled or uncoupled in the absence of applied current. For a smaller leak $(L=2)$, an intermediate state occurs in which only a small maintained current is required for bistability. Increases in the leak in cell 1 decrease the voltage changes in cell 1 associated with changes in coupling, but have little effect on the voltage changes in cell 2 .

A similar set of relations obtains for the leak in cell 2 (Fig. 8, left column; shown for negative transjunctional voltages). Increase in the leak shifts the $\mathrm{V}$-I relations to the right, but in this case the range of currents permitting bistability is narrowed. With a sufficiently large leak, the range of bistability can include zero current $(L=4)$ and the cells can be either stably coupled or uncoupled without current application as for a similar lcak in cell 1 . Coupling and uncoupling are associated with appropriate shifts in resting potential and describe the phenomena shown in Figure 7 . With a lesser leak $(L=2)$, the region of bistability does not include zero current but can be entered with a small current. Cells uncoupled by such a current presumably would recouple gradually on termination of the current, as in Figure $7 \mathrm{C}$. Increases in the leak in cell 2 decrease the voltage changes in cell 2 associated with changes in coupling but have little effect on the voltage change in cell 1 .

The calculations are shown only for currents for which transjunctional voltages remain of a single polarity; symmetric regions of bistability exist for currents which produce transjunctional voltages of the opposite polarity. When a leak is present, the cells can exhibit a form of rectification. For example, if cell 1 is leaky, depolarizing current pulse may uncouple the cells, while a hyperpolarizing current pulse of the same magnitude will not uncouple them (although a much larger hyperpolarizing pulse would do so). Unlike transmission at rectifying electrotonic synapses, the rectification develops slowly because of the slow changes in junctional conductance.

When the internal resting potentials of the two cells differ $\left(E_{1}\right.$ and $E_{2}$ in Fig. $1 B$ ), intrinsic currents are generated and the V-I relations are simply translated with respect to the axes without change in shape or scale. If the resting potential of cell 2 is increased with respect to that of cell 1 , the V-I relations are moved leftward along the current axis, reducing the depolarizing current required for bistability and increasing the required hyperpolarizing current. Intrinsic currents arising from electrogenic pump activity result in similar translation of the V-I relations.

Unequal resting potentials and the presence of leakage conductances can have additive effects on the V-I relations. Combination of appropriate differences in resting potentials and leakage conductances can cause cells to exhibit bistability with zero current when either difference alone does not do so.

These studies show that membrane properties which generate or change intrinsic currents can alter the stability of states of coupling and the thresholds for the transitions between them.

Parametric studies. In order to gain a qualitative appreciation of how differences in the junctional and nonjunctional conductances affect bistability of coupling, steady state V-I relations were calculated for various values of the maximum junctional conductance, $g_{\max }$, and the nonjunctional conductances $g_{1}$ and $g_{2}$ (Fig. 9; equations 16 to 18). The effects of these conductances on the shapes of the V-I relations are treated quantitatively in the "Appendix."

With all other conductances constant, for decreasing $g_{\max }$, the region of bistability occurs at smaller applied currents, becomes narrower, and disappears at some critical value. For smaller values of $g_{\max }$, the system is stable at all levels of applied current; membrane potentials and coupling change as a smooth function of current. The effects of the changes in the region of bistability are that 
with decreasing $g_{\max }$, stable changes in coupling can occur at smaller levels of applied current.

Decreasing $g_{1}$ moves the region of bistability leftward and narrows it, while increasing the change in membrane potentials when the cells uncouple and recouple. Increase of $g_{2}$ has qualitatively similar effects except that the input conductance of the system is increased as well.
Coupling within populations of cells. Coupling patterns during applied current pulses were studied in a small population of cells. In the experiment of Figure 10, three blastomeres within a group containing fewer than ten were impaled with voltage recording electrodes and current was passed into cell 1 with a separate microelectrode. Initially, all cells were coupled (pulse 1). As the
LEAK IN CELL 2
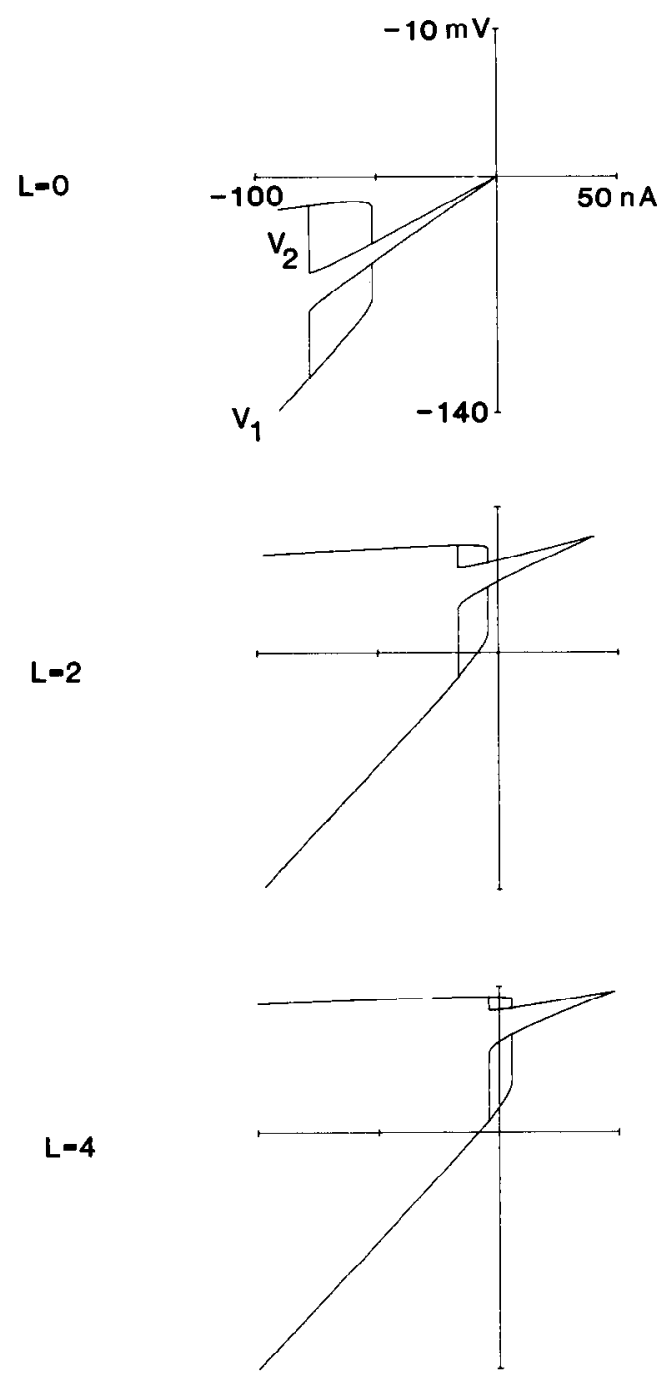

LEAK IN CELL 1
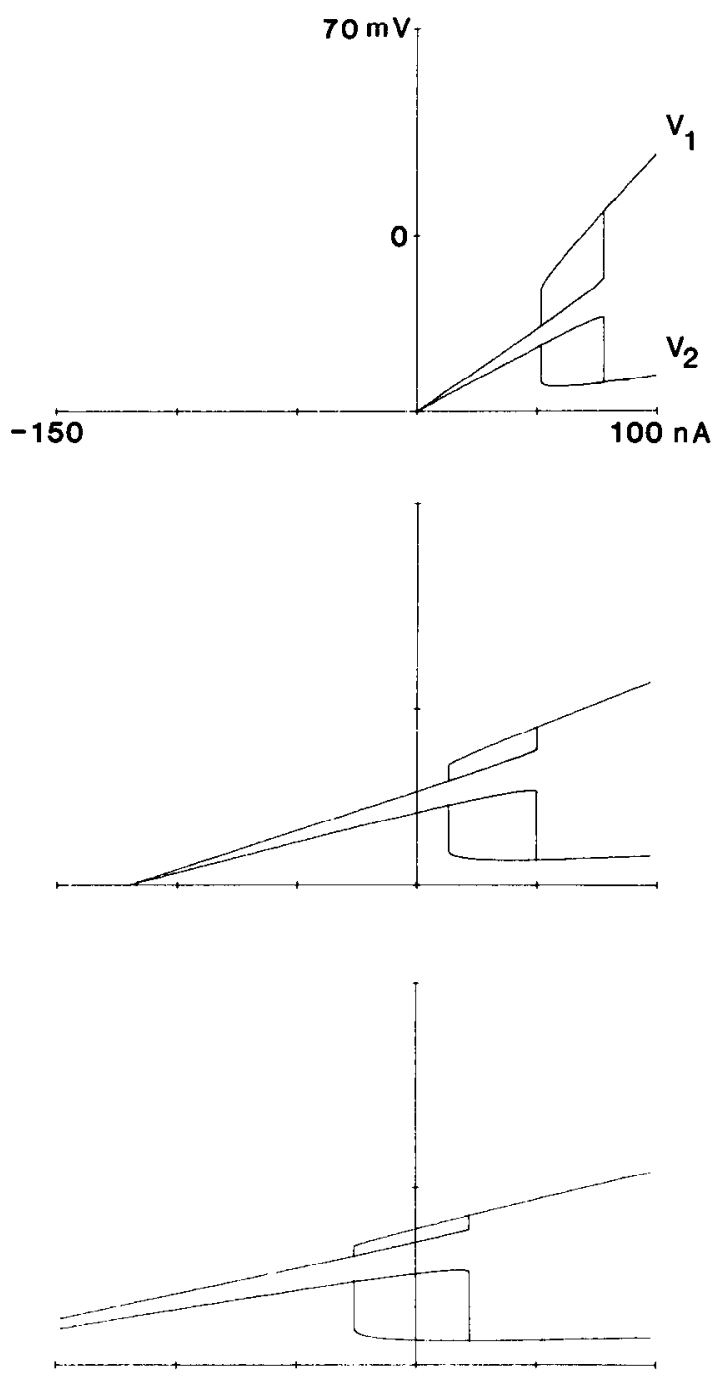

Figure 8. Calculated effects of nonjunctional leakage conductances on the V-I relations of coupled pairs of cells. Calculated VI relations are shown for various leakage conductances in cells 1 and $2(L=0,2$ and $4 \mu \mathrm{mho})$ with $g_{1}=g_{2}=1.0 \mu \mathrm{mho}$ and $g_{\text {max }}=$ $4 \mu \mathrm{mho}$ and $E_{\mathrm{l}}$ and $E_{2}$ equal to $-60 \mathrm{mV}$ (equations 16 to 18 ). For leakages in each cell, the calculations are shown only for currents for which transjunctional voltages remain of a single polarity (cell 1 more positive for leak in cell 1 and more negative for leak in cell 2); regions of bistability symmetric about the point where $V_{j}=0$ exist for currents which produce transjunctional voltages of the other polarity. Right column, Leakage conductance in cell 1 . The V-I relations without a leakage conductance $(L=0)$ are in the upper plot. The effect of adding a leak in cell 1 is to shift the V-I relations to the left and enlarge the current range of bistability. When there is a sufficiently large nonjunctional leak in cell $1(L=4)$, the range of bistability includes zero current, and the cell pair can be either stably coupled or uncoupled in the absence of applied current. With a lesser leak in cell $1(L=2)$, the region of bistability does not include zero current but could be reached by application of a small depolarizing current. Left column, Leakage conductance in cell 2 . The V-I relations without a leakage conductance in cell $2(L=0$; upper plot) are symmetrical with regard to the plot for no leak in cell 1 . Leakage conductance in cell 2 shifts the V-I relations to the right and narrows the range of currents which permit bistability. With a sufficiently large leak, the range of bistability includes zero current $(L=4)$ and the cells can be either coupled or uncoupled without current application. The conditions are associated with appropriate shifts in membrane potential and describe the phenomena shown in Figure 7. With a lesser leak $(L=2)$, the region of bistability does not include zero current but can be entered with a small hyperpolarizing current. 
A

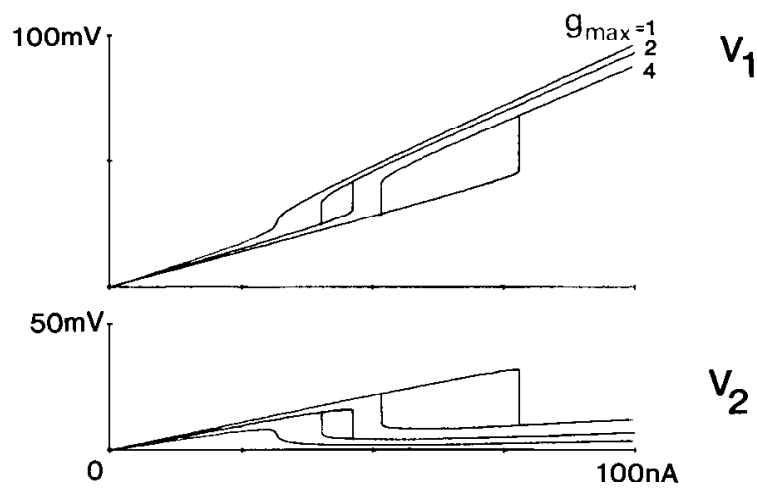

B

C

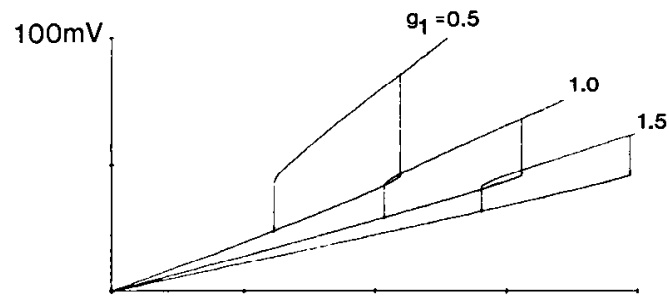

$100 \mathrm{mV}$
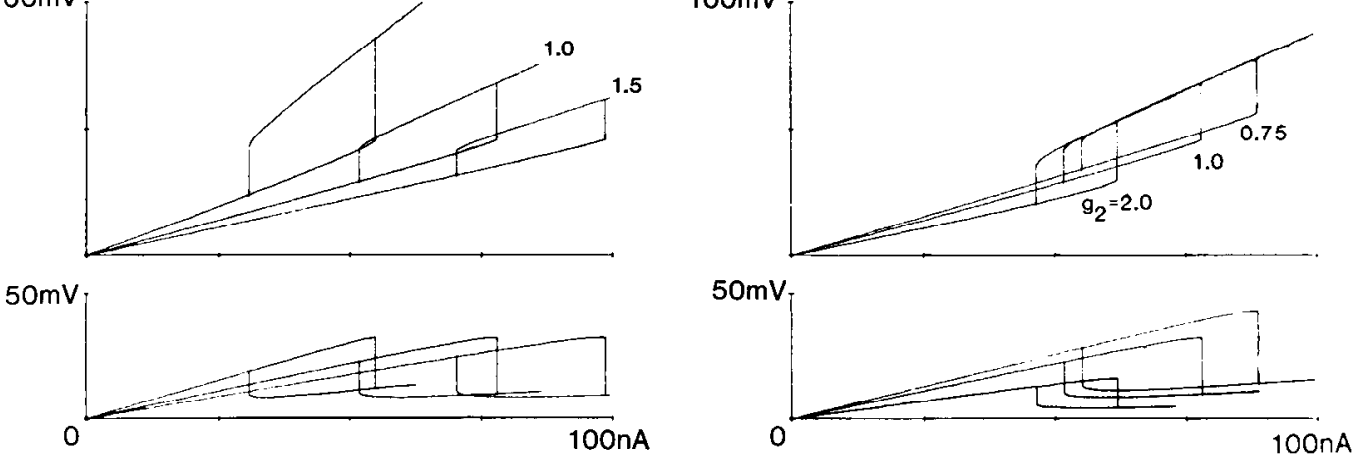

Figure 9. Effects of variation in $g_{m a x}, g_{1}$, and $g_{2}$ on the steady state V-I relations of coupled pairs of cells. The steady state V-I relations were calculated for conditions in which the maximum conductance $\left(g_{\max }\right)$ and the nonjunctional conductances $\left(g_{1}\right.$ and $g_{2}$ ) of the cells were varied (equations 16 to 18 ). Unless otherwise specified, $g_{\max }=4 \mu \mathrm{mho}, g_{1}=g_{2}=1 \mu \mathrm{mho}$, and $E_{1}=E_{2}=-60$ $\mathrm{mV}$. $A$, Variation in $g_{\max }$. The V-I relations were calculated for $1.0<g_{\max }<5.0 \mu \mathrm{mho}$. For decreasing $g_{\max }$, the region of bistability occurs at smaller applied currents, becomes narrower, and disappears for some value of $g_{\max }$ between 1.0 and $2.0 \mu$ mho. For all values of $g_{m a x}$ sinaller than this value, the system is stable at all levels of applied current; membrane potentials and coupling change as a smooth function of current. For $g_{\max }$ increasing beyond this value, the region of bistability subtends successively larger ranges of current. For sufficiently large $g_{\max }$ (greater than a value between 3 and $4 \mu \mathrm{mho}$ ), the system becomes bistable in voltage as well as current. $B$ and $C$, Decreasing $g_{1}$ moves the region of bistability leftward and narrows it, while increasing the change in membrane potentials when the cells uncouple and recouple. Increase of $g_{2}$ has qualitatively similar effects except that the input conductance of the system is increased as well.

current pulses increased in size and duration, cell 3 uncoupled from cell 2 (pulses 2 to 4 ). With larger pulses, cell 2 uncoupled from cell 1 and cells 2 and 3 apparently recoupled (pulses 5 and 6 ). These data demonstrate that the boundaries of coupling within a group of cells can be altered by applied current.

This interpretation is supported by data in Figure 11 from the same cells and by the calculations shown in Figure 12. In Figure 11A, a large current pulse uncoupled cells 2 and 3 from cell 1 (pulse 1). During an identical pulse to cell 1, a small current pulse delivered to cell 3 through the recording microelectrode ( pulse 2) revealed marked coupling of cell 3 to cell 2, even when cell 2 was uncoupled from cell 1 . During a larger pulse in cell 3 ( pulse 3 in cell 1), cell 3 became at least partially uncoupled from cell 2 .

These cells later exhibited long-lasting uncoupling (Fig. 11B) similar to that shown for a pair of cells (Fig. $7, A$ and $B$ ). Strong hyperpolarization of cell 1 (pulse 1 ) uncoupled it from cells 2 and 3 and caused cell 3 to become stably uncoupled, while cells 1 and 2 recoupled following termination of the applied current. These changes in coupling are demonstrated at the onset of pulse 2. Application of a hyperpolarizing pulse to cell 3 during this pulse recoupled it to cell 2 , demonstrating bistability, and all three cells were coupled at the onset of pulse 3 .

The complexities in the multicellular system preclude simple analysis. For example, it is likely that coupling of cells 1 and 3 was not exclusively via cell 2 . Nonetheless, it is clear that cell groups can be partitioned differently depending on the site and strength of current application and at least some of these partitionings can be stable. Also, uncoupling within populations of cells can be virtually complete (Fig. $11 B$ ), unlike the results with pairs of cclls, probably because there can be more than one low conductance junction in series between the cells from which one is recording. In Figure $11 B$, quite small changes in steady state potentials were associated with stable uncoupling. Larger changes presumably occurred 
at the affected junctions, again indicating the difficulty of analysis in multicellular (or nonisopotential) systems.

Calculations of steady state V-I relations for three linearly arranged cells. The V-I relations of three linearly arranged coupled cells were calculated according to equations 19 to 21 for parameters chosen to demonstrate sequential uncoupling and recoupling with rising and falling current (Fig. 12). With increasing current, first cell 3 uncouples from cell 2 , then cell 2 uncouples from cell 1 and recouples to cell 3 . With decreasing current, cells 2 and 3 remain coupled throughout and cell 1 recouples to cell 2 at a current below that where uncoupling first occurred with increasing current. Other sequences of uncoupling and recoupling are possible; in these calculations the values of junctional and nonjunctional conductances were chosen in order to demonstrate the sequences indicated by the data shown in Figures 10 and 11.

These data and calculations show that in the case of several cells coupled via voltage-dependent junctional membranes, cells can be stably coupled to one another and stably uncoupled in groups, where the boundaries between the groups depend on the value of applied current. Although not demonstrated here, intrinsically generated curents or differences in resting potentials can create boundaries in similar ways in the absence of applied current.

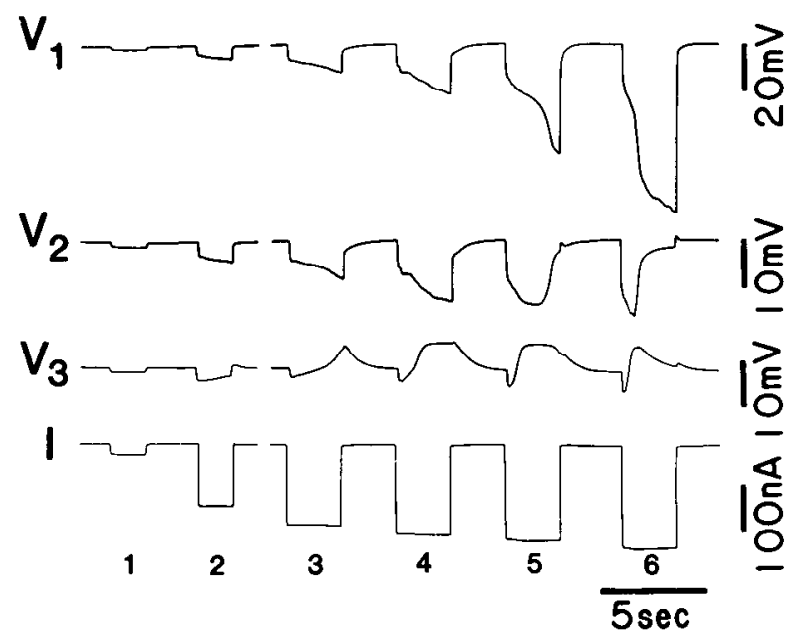

Figure 10. Uncoupling within a population of cells during applied current pulses. Three blaslomeres within a group containing fewer than ten were impaled with voltage recording electrodes $\left(V_{1}, V_{2}\right.$, and $\left.V_{3}\right)$ and current was passed into cell 1 with a separate microelectrode. A small current pulse ( pulse 1) showed close coupling between cells 1,2 , and 3 . During a larger pulse (pulse 2), cell 3 began to uncouple from cells 1 and 2 . Slightly longer and larger current pulses caused cell 3 to uncouple from cells 1 and 2 and to depolarize to a new membrane potential until the end of the pulse (pulses 3 and 4). Still larger current pulses ( pulses 5 and 6 ) produced a large increase in the input resistance of cell 1 and uncoupled it from cell 2 as well as cell 3 . In the latter part of pulses 5 and 6 , the membrane potential of cell 3 recovered toward its previous level, which suggests that cell 3 recoupled to cell 2 soon after cell 2 uncoupled from cell 1 .
A

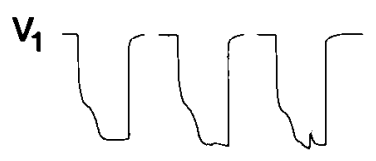

$\left.\left.v_{2}\right] \sqrt{\wedge}\right] \sqrt{ }^{\curlywedge}$

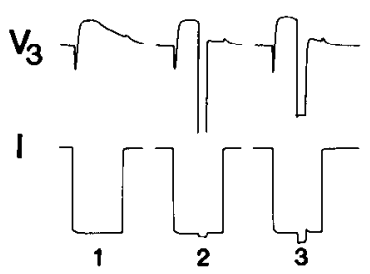

B
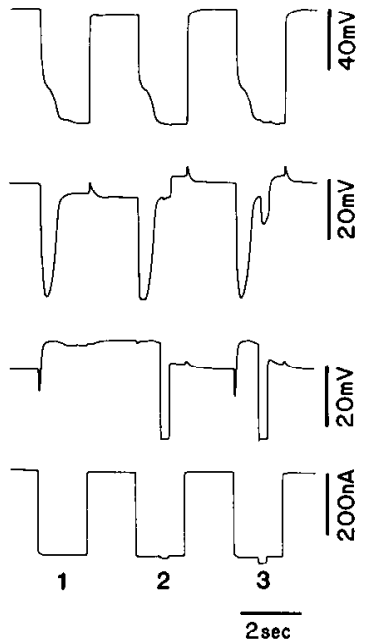

Figure 11. Uncoupling, recoupling, and bistability within a population of cells. A, Pattern of recoupling during movement of coupling boundaries with applied current pulses. During pulse 1 , cell 3 uncoupled from cells 1 and 2 , and then cell 2 uncoupled from cell 1. During a second pulse in cell 1 of the same size, a small current pulse was delivered to cell 3 through the recording microelectrode. This superimposed pulse revealed coupling of cell 3 to cell 2 , but very little coupling to cell 1 . During a third pulse in cell 1, a larger brief pulse in cell 3 caused a potential in cell 2 that declined during the brief pulse. This decline is likely to represent recoupling of cells 2 and 3 , since the potential in cell 2 went to a more depolarized level immediately after the pulse. The positively directed transient in cell 1 at the end of the brief pulse in cell 3 indicates that partial recoupling of cell 1 had occurred during the pulse. $B$, Bistability of coupling. Strong hyperpolarization of cell 1 ( pulse 1) uncoupled it from cells 2 and 3 . Following this pulse, cell 3 attained a stable, more depolarized resting potential and cells 1 and 2 remained somewhat hyperpolarized. Cells 1 and 2 recoupled, while cell 3 remained uncoupled as indicated by the initial effects of pulse 2. The completeness of uncoupling of cell 3 from the other cells is shown by the lack of change in $V_{3}$ at the onset of pulse 2. Pulse 2 also uncoupled cells 1 and 2 . A brief, small hyperpolarizing pulse in cell 3 during pulse 2 evidently recoupled cells 2 and 3 and both membrane potentials became slightly depolarized from their initial values. At the end of pulse 2, all three cells recovered their initial membrane potentials. Pulse 3 showed that all three cells had recoupled and produced a sequence of uncoupling like that during the first pulse. A larger brief pulse in cell 3 again appeared to recouple cells 2 and 3 with little effect on the potential in cell 1 .

\section{Discussion}

Phenomenology of voltage-sensitive coupling. The effects of applied current on coupling between Ambystoma blastomeres are predicted by voltage dependence of the junctional conductance. Pairs of amphibian blastomeres can have V-I relations with regions of negative slope that lead to bistability of coupling with regenerative transitions between stable coupled and uncoupled states. Such cells show hysteresis when increasing and decreasing currents are applied and thus their state of coupling can depend on previous history. 
The criteria for existence of bistability are considered in the "Appendix." The tendency toward bistability is greater when voltage dependence of junctional conductance is steeper and when $g_{\min }$ is smaller. The tendency toward bistability is less when nonjunctional conductances are greater. The importance of bistability for control of intercellular communication is that brief currents or changes in nonjunctional membranes can trigger transitions between stable states of coupling. Except possibly when cells are very close to threshold, the change in junctional conductance during a transition is nearly the entire difference between $g_{\max }$ and $\boldsymbol{g}_{\min }$. When coupling is not bistable, comparable changes in junctional con-

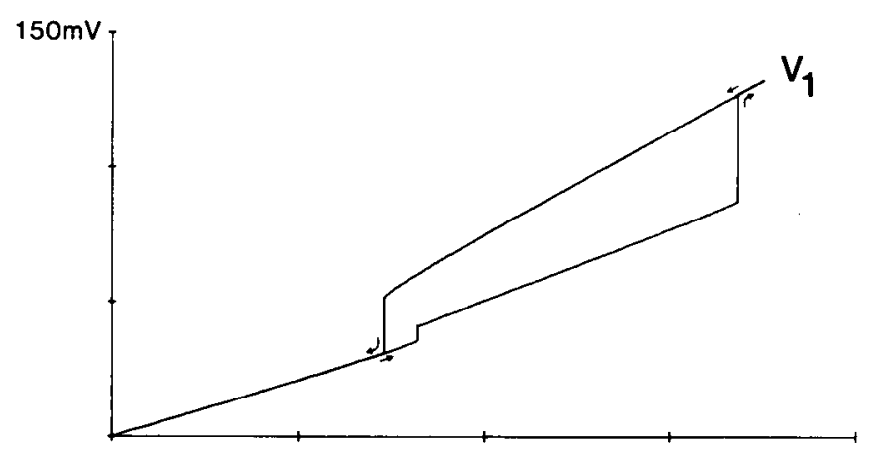

$100 \mathrm{mV}$
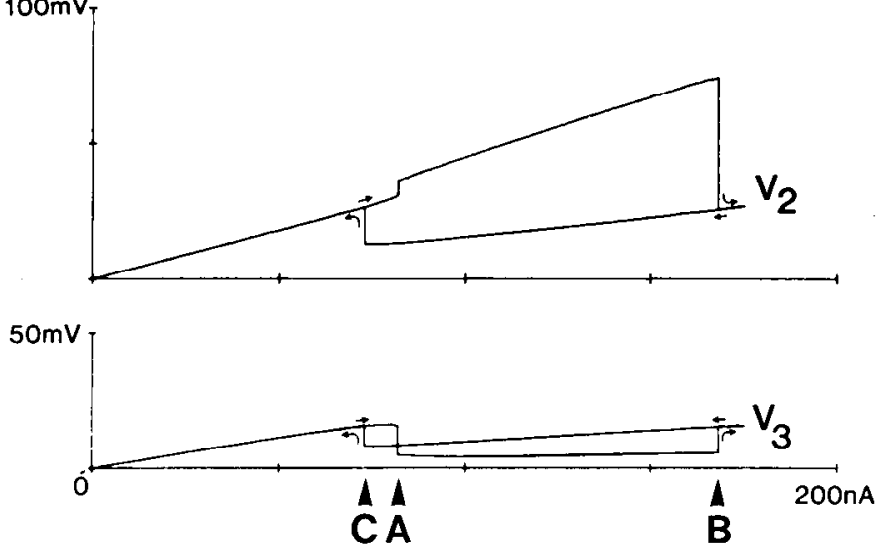

Figure 12. Calculation of steady state V-I relations for three linearly arranged cells. Values were calculated according to equations 19 to 21 for conductances chosen to demonstrate sequential uncoupling and recoupling with rising and falling current: $g_{1}=g_{2}=g_{3}=1 \mu \mathrm{mho}, g_{j 1}=10 \mu \mathrm{mho}$, and $g_{j 2}=2$ $\mu \mathrm{mho}$. Moderate polarization of cell 1 increases the voltages of all three cells along slopes defining their input and transfer resistances. At about $75 \mathrm{nA}$ with these parameters, $V_{1}$ and $V_{2}$ show a small upward shift, while $V_{3}$ suddenly drops (point $A$ ). At this point, cells 1 and 2 are coupled to each other, and cell 3 is uncoupled from cell 2. With application of about $150 \mathrm{nA}$ to cell $1, V_{1}$ abruptly increases and $V_{2}$ decreases (point $B$ ). $V_{3}$ shows a slight increase. At this point, cells 2 and 3 are coupled to each other and cell 1 is uncoupled from cell 2 . With decreasing current, cells 2 and 3 remain coupled, and when the applied current is just below a value of $75 \mathrm{nA}$, cell 1 recouples to cell 2 ( point $C$ ). These calculated V-I relations show that in the case of several cells coupled via voltage-dependent junctions, cells can be stably coupled to one another and stably uncoupled in groups, where the boundaries between the groups are plastic and depend on the level of applied current. ductance can occur under appropriate conditions but are graded.

Even without extrinsically generated transjunctional current, current from intrinsic sources can be adequate to uncouple the cells and to maintain them in an uncoupled state. Steady state and transient intrinsic currents could arise from differences in nonjunctional membrane properties such as ionic selectivity and electrogenic pump activity. If coupling is bistable over a narrow region, the uncoupling and recoupling can occur with relatively small perturbations in intrinsic or applied currents near this region. A wide region of bistability indicates that if a transition between states in one direction is produced by a small perturbation, a relatively large perturbation is required to return the coupling to its initial state.

The ability to model the kinetic and steady state properties of pairs of coupled cells has allowed us to attribute bistability to the junctional membrane. It also permits study of how the properties of the junctional and nonjunctional membranes affect coupling behavior. Significant findings include the following.

1. Cells can be bistable in coupling due to applied or intrinsic currents.

2. Brief currents of either extrinsic or intrinsic origin can trigger transitions between stable states of coupling.

3. The threshold for uncoupling can be much lower for one polarity of current than for the other.

Occurrence of voltage-dependent junctional conductance. Voltage dependence of junctional conductance has been observed between blastomeres of three amphibian genera, Ambystoma, Xenopus and Rana (Spray et al., 1981a). Nonlinear junctional conductance was suggested earlier as an explanation for occasional findings in pairs of Xenopus blastomeres (Slack and Warner, 1975). Similar voltage-sensitive coupling recently was found in later stage Xenopus embryos between developing RohonBeard neurons (Spitzer, 1980, 1982). Gap junctions between early teleost (Fundulus) blastomeres are also voltage dependent but to a lesser degree than in amphibian embryos (White et al., 1982). Records from Limulus ommatidia bear a striking resemblance to those of the present study, and voltage-dependent junctional conductance may be present between retinula cells (Smith et al., 1965; Smith and Baumann, 1969).

Other forms of voltage-sensitive coupling have been seen in other systems. When coupling between squid blastomeres is reduced by cytoplasmic acidification, junctional conductance can be increased by large transjunctional voltages, which appears to represent a different mechanism than that found in amphibian embryos (Bennett et al., 1982). The cells of the salivary gland of Chironomus larvae uncouple with depolarization and recouple with hyperpolarization (Rose, 1970; Socolar and Politoff, 1971). The uncoupling does not require a transjunctional voltage (Obaid and Rose, 1981).

If cells coupled by voltage-dependent junctions had different resting potentials, the junctions could appear to rectify in a manner similar to those of the crayfish giant motor synapse (Furshpan and Potter, 1959), the sensorymotoneuron synapse in the leech (Nicholls and Purves, 1970), and the giant fiber-motoneuron synapse of the 
hatchetfish (Auerbach and Bennett, 1969). However, rectification at these synapses occurs in the submillisecond range, in contrast to all of the previous examples where time constants are in the range of hundreds of milliseconds. Thus the mechanisms at these synaptic and nonsynaptic junctions are likely to be different.

Although there are few known examples of junctional voltage sensitivity like that in amphibian embryos, the relatively slow changes in junctional conductance could easily have been missed in many studies. Furthermore, changes of this kind can be obscured by lack of isopotentiality within cells as well as by the requirement for a significant transjunctional voltage, which can be difficult to achieve experimentally in closely coupled cells.

Implications for intercellular signaling during development. The plasticity of coupling mediated by voltagedependent junctions and its sensitivity to differences in resting potential and to applied or intrinsic current provides an attractive mechanism by which patterns of intercellular communication may be established and changed (for reviews, see Bennett et al., 1981a,b; Spray et al., 1982c). An experimental demonstration of this possibility is given in the recordings from a group of cells in which the boundaries of coupling changed as a function of applied current (Figs. 10 and 11). Apparently, complex patterns occurred in even this small group of cells.

The presence of voltage-dependent coupling between blastomeres may allow significant changes in intercellular communication, transiently or long term, by changes in any of a number of cellular parameters. For example, a gradient of a regulatory substance within an embryo or a portion of an embryo (see Wolpert, 1969) could produce a gradient of resting potentials by an alteration of membrane selectivity, of ion concentrations through exchange or transport, of electrogenic pumping, or of any basic cellular function (such as metabolic rate) which affects these factors. A gradient of resting potentials across a population of cells within an embryo would generate steady state intrinsic currents. In response to a transient current or a change in the steady state current, threshold could be reached and a population of cells could be regeneratively and stably uncoupled. In this way, voltagedependent junctions could convert a continuous feature of an embryonic tissue, a gradient of resting potentials, into a discontinuous feature; that is, a boundary of interrupted coupling between two portions of the tissue.

There are few reports from which to conclude whether or not such changes occur naturally. In Xenopus embryos, it has been shown that developing neural ectoderm undergoes a strophanthidin-sensitive hyperpolarization with respect to the lateral ectoderm to which it is coupled and from which it differentiates (Blackshaw and Warner, 1976). Coupling between the two populations of cells disappears at the time of neural lube closure. It is possible that voltage sensitivity of the junctions plays a part in the establishment of a coupling boundary between these two populations of cells. However, in isotonic potassium plus strophanthidin the cells are uniformly depolarized, but development proceeds normally (Messenger and Warner, 1979). Thus voltage-dependent uncoupling apparently is not necessary at this stage.

Any developmental change which alters coupling would tend to alter the conditions required for uncoupling and the resulting patterns of intercellular communication. Junctional conductance can be altered by small changes in intracellular pH (cf. Spray et al., 1981b, 1982a), relatively large changes in intracellular calcium ion (Spray et al., 1982b), or presumably by changes in the number of junctional channels (which can be under hormonal control; cf. Merk and McNutt, 1972; Elias and Friend, 1976). Changes in nonjunctional conductances also can affect coupling (cf. Spira et al., 1980). In addition, voltage-sensitive coupling may be present between some cells and not others at the same stage of development (Spitzer, 1980, 1982; although it is difficult to exclude geometrical factors in this case). The degree of coupling, measured either directly or inferred from the occurrence of gap junctions, is known to change in a number of instances at specific, apparently developmentally relevant times (cf. Bennett et al., 1981a,b; Goodman and Spitzer, 1979; Raper and Goodman, 1982). Whether voltage-dependent changes in junctional conductance have any influence on the cellular mechanisms that control the incidence of gap junctions is unknown. Voltage-dependent uncoupling can remain reversible for many minutes, but longer periods have yet to be tested.

Probably the best measure of intercellular electrical signaling by voltage dependence of junctional conductance is the difference between the postjunctional potentials before and after the change in junctional conductance. A parametric analysis of the factors which determine this difference is given in the "Appendix." The role of electrical signaling during development remains to be established, but, in addition to possible effects of change in transmembrane potential in influencing cell properties, there may be electrophoresis of cytoplasmic constituents and of membrane-bound macromolecules along the plasma membrane surface (Jaffe, 1981; Poo et al., 1979).

One must be cautious in inferring degree of chemical coupling from degree of electrical coupling and vice versa. The electrical coupling coefficient (equation 3 ) is a function of junctional and nonjunctional conductances. One can define an analagous chemical coupling coefficient where leak or metabolism of a substance corresponds to nonjunctional conductance and junctional permeability corresponds to junctional conductance. For a molecule that is not metabolized and that can cross the junctional membranes but not the nonjunctional membrane, the chemical coupling coefficient is unity, while the electrical coupling coefficient can be high or low. Conversely, for a rapidly metabolized molecule or one which readily permeates nonjunctional membrane the chemical coupling coefficient can be less than the electrical coupling coefficient; of course, for a molecule that does not permeate the junctions, the chemical coupling coefficient is zero.

For chemical coupling the analogue of membrane capacity is cell volume. For the common fluorescent tracers, intercellular equilibration time constants can be minutes to hours (cf. Bennett et al., 1978). The great discrepancy possible between electrical and chemical time constants must be considered in comparing the two kinds of measurement.

Nonisopotentiality within cells also provides possibilities for discrepancies between chemical and electrical 
coupling. If cells are joined by way of thin processes of axial resistance too high to permit measurable electrical coupling, they can still be closely coupled chemically. Conversely, if there were a large access resistance to the junctions or if the postjunctional cell were of low conductance, changes in electrical coupling coefficient due to voltage dependence of junctional conductance could be small when junctional conductance, permeability, and the chemical coupling coefficient were markedly reduced. Furthermore, the site at which the electrical communication is measured may be quite distinct from the site at which the intercellular message acts.

In correlating changes in electrical and chemical coupling one should know whether selectivity changes as conductance changes. If the decrease in junctional conductance with transjunctional voltage is due to decrease in the mean open time of junctional channels of constant unitary conductance, selectivity will not change; if the unitary conductance of the junctional channels decreases, the selectivity presumably will change. Single channel studies on the voltage-sensitive channels of nerve and muscle indicate that reduction of the macroscopic conductance generally is due to decrease in channel open time, not in the magnitude of the unitary conductance (cf. Neher and Steinbach, 1978; Horn et al., 1981). If the same is true of the voltage-dependent changes in junctional conductance, the intercellular flux of permeant molecules would be approximately proportional to the junctional conductance. The residual conductance $g_{\text {min }}$ would represent a population of channels not affected by voltage and of unchanged selectivity. Although we found that in amphibian blastomeres a decrease in junctional conductance decreased the flux of Lucifer Yellow (ion $M_{\mathrm{r}}$ 443), our methods were not sufficiently sensitive to determine whether the conductance $g_{\min }$ allowed any transjunctional dye movement (Spray et al., 1979). A number of studies utilizing fluorescent probes suggest that junctional selectivity can change either developmentally or during chemical treatments (cf. Rose and Loewenstein, 1975; Goodman and Spitzer, 1979). In no case, however, has there been a quantitative correlation between decrease in junctional conductance and permeability. When junctional conductance is reduced, an apparent change in selectivity can result from reduction of intercellular tracer flux to a nonzero level below the limit of detection.

In view of the single channel studies, and in the absence of convincing evidence to the contrary from tracer studies, it is reasonable to assume that the changes in junctional conductance during voltage-dependent uncoupling are not accompanied by changes in selectivity of the junctional channel. If, however, $g_{\min }$ is due to junctional channels of lesser permeability, chemical coupling for larger molecules could be completely abolished while significant electrical coupling persisted.

The presence of voltage-controlled intercellular pathways in developing tissue provides a new mechanism for the regulation of this intimate form of cellular communication during development. Gap junctions have been shown to mediate the intercellular transfer of metabolically important molecules (Pitts and Finbow, 1977) and of intracellular messengers (Lawrence et al., 1978). The ubiquity of electrotonic coupling in developing tissues has prompted the suggestion that developmental processes are in some manner influenced by the chemical coupling which the electrical pathway mediates (Furshpan and Potter, 1968). Complex biochemical patterning of tissues can arise from the passive spread of interacting molecular signals (Gierer, 1977). It is possible that voltage-controlled uncoupling and/or recoupling play essential roles in defining the pattern of cellular interactions necessary for proper development and differentiation.

\section{Appendix}

\section{Voltage-dependent conductance and $V$-I relations}

In this appendix we consider how voltage dependence of junctional conductance affects V-I relations and electrotonic coupling of amphibian blastomeres. Initially, we define the properties of an idealized voltage-dependent conductance that are required for its $\mathrm{I}-\mathrm{V}$ relation to have a region of negative slope. We then relate these properties to characteristics of the amphibian junctional conductance. Finally, we examine the effects of nonjunctional conductances on the V-I relations and optimization of conductance parameters for control of intercellular communication.

Idealized voltage-dependent conductances. Assume a voltage-dependent resistance, $r$, or conductance $g=1 / r$. Starting with Ohm's law $I=V / r=V g$ and differentiating we obtain:

$$
\begin{aligned}
\frac{d I}{d V} & =\frac{1}{r}-\frac{V}{r^{2}} \frac{d r}{d V} \\
& =g+V \frac{d g}{d V}
\end{aligned}
$$

The slope $d I / d V$ is negative if:

$$
\frac{d r}{d V}>\frac{r}{V} \text { or }-\frac{d g}{d V}>\frac{g}{V}
$$

Thus, where $r$ increases with $V$ at a rate greater than $r / V$ or $g$ decreases with $V$ at a rate greater than $g / V$, there is a negative slope in the $\mathrm{I}-\mathrm{V}$ relation (and the V-I relation as well).

For illustration consider an arbitrary resistance that increases linearly with voltage from $r_{\min }$ to $r_{\max }$ starting at some voltage $U_{1}$. Figure A1 shows the $r-V, g-V$ and I$\mathrm{V}$ relations for such a resistance with rates of increase (s) between 0.5 and 4 times the value $r_{\min } / U_{1}$. The I-V plots (Fig. A1C) show hyperbolic transitions between two linear regions whose slopes are determined by $r_{\text {min }}$ and $r_{\max }$. For $s=0.5, d r / d V=r_{\min } / 2 U_{1}$ and it follows from inequality (2) that the slope of the I-V relation is everywhere positive. For $s=2$ and $s=4$ there are negative slope regions when $d r / d V$ becomes greater than $r_{\min } / U_{1}$. For the critical value $s=1, d r / d V=r_{\min } / U_{1}$ and the $\mathrm{I}-\mathrm{V}$ relation is a horizontal region (degenerate hyperbola). Graphically, one sees that irrespective of the shape of the $r$ - $V$ relation, the slope of the I-V relation is negative at each voltage for which the slope at the corresponding point of the $r-V$ relation is greater than the slope of the straight line drawn from the origin to that point.

The I-V relation for $s=2$ is redrawn as a V-I relation in Figure $\mathrm{A} 1 D$ to correspond to the V-I relations in the text. The hysteresis that would be exhibited during rising 
and falling ramps of current is indicated by dashed lines and arrows; the voltage is bistable between $I_{1}$ and $I_{2}$ which are the rheobasic currents for transitions between low and high resistance states (or high and low conductance states). The ratio of upper and lower voltages at threshold for uncoupling, $U_{2} / U_{1}$, depends on the ratio of $r_{\max }$ to $r_{\min }$. It may be seen by inspection of Figure A1 $A$ and $C$, that threshold current for decrease in resistance $\left(I_{2}\right.$ in Fig. $\mathrm{A} 1 D$ ) decreases with both the steepness (larger $s$ ) and magnitude of the increase in $r$, while the threshold for increase in resistance $\left(I_{1}\right)$ remains constant. Thus these changes in voltage sensitivity increase the size of the region of bistability in terms of the fraction of threshold current, $\left(I_{1}-I_{2}\right) / I_{1}$. The threshold voltage $U_{3}$ for regenerative change from high to low resistance condition is greater than the threshold voltage, $U_{1}$, for increase in resistance but converges to it as $s$ increases. For $s=0.5$, the relations are single valued in both current and voltage (see Fig. $\mathrm{A} 1 \mathrm{C}$ ). For this case, there is no bistability or threshold, although the change in resistance at the onset of a sufficiently large current pulse is regenerative.

Corresponding arguments can be made from the $g-V$ relations (Fig. $A 1 B$ ) but they are algebraically more untidy. For a linear increase in $r$, the critical rate of decrease of conductance is a hyperbola $g=g_{\min } U_{1} / V$. At any point in a $g-V$ relation where $g$ decreases more rapidly than the corresponding hyperbola, the slope of the $\mathrm{I}-\mathrm{V}$ relation is negative.

A negative slope in the I-V relation also can result from a conductance that decreases as a function of current; the relations are exactly the same as those for Figure Al except that $V$ and $I$ are interchanged and $g$ and $r$ are interchanged. The resulting I-V relations can be bistable in current as a function of voltage over a
A

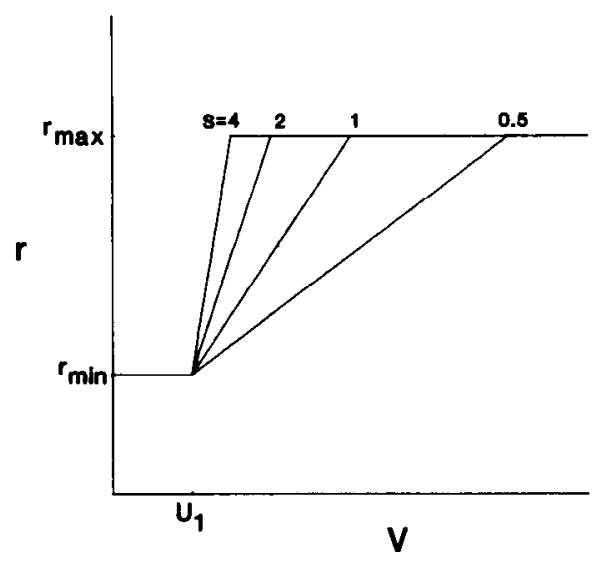

B

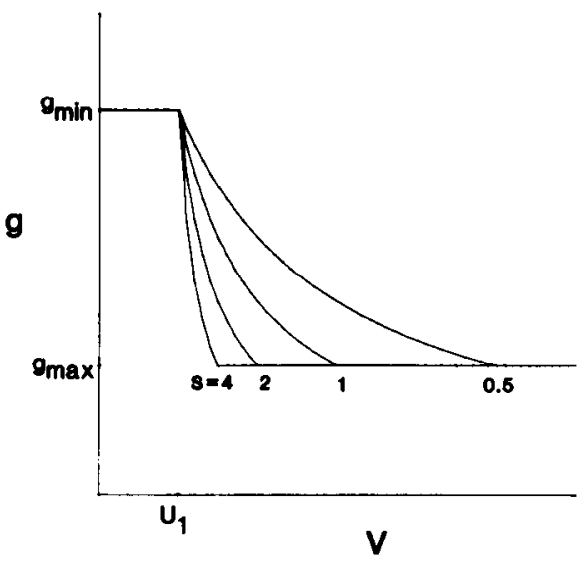

C

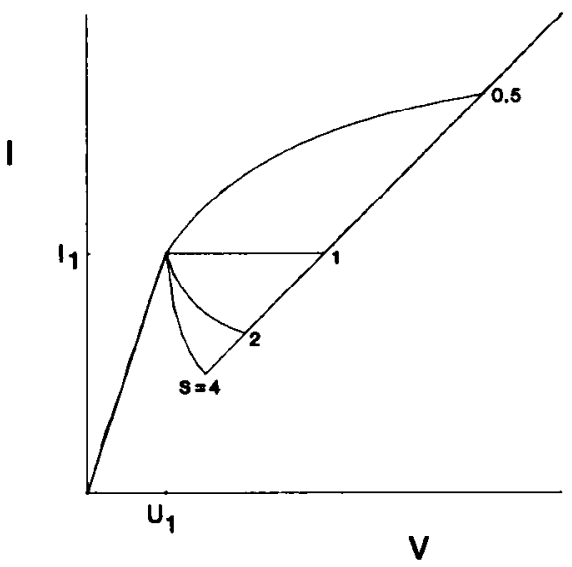

D

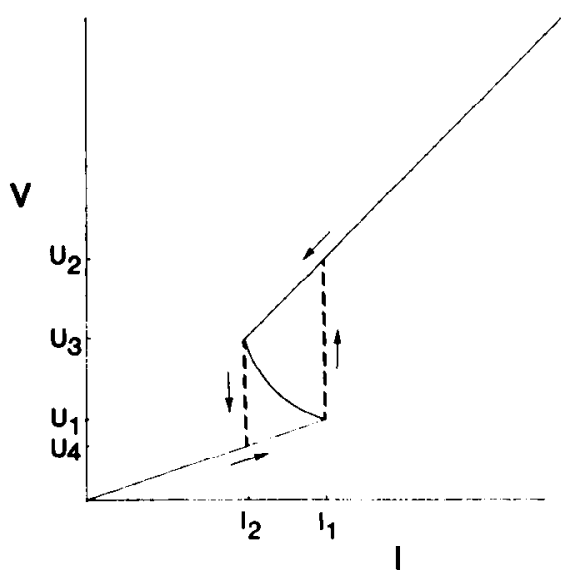

Figure A1. Criteria for a negative slope region to appear in the I-V relation of a voltage-dependent resistance or conductance. $A$, A resistance increasing linearly with voltage from $r_{\min }$ to $r_{\max }$ starting at the voltage $U_{1}$ with slope equal to $s r_{\min } / U_{1}$ where $s$ $=0.5,1,2$, and $4 . B$, The corresponding relations between conductance and voltage. Conductance decreases from $g_{\max }$ to $g_{\min }$ along a segment of the hyperbola $g / g_{\max }=1 /\left(s V / U_{1}-s+1\right)$. C, The corresponding I-V relations. The portions between the two straight lines of positive slope are segments of the hyperbola $I / I_{1}=V / U_{1} /\left(s V / U_{1}-s+1\right)$ where $I_{1}$ is the current at $U_{1}$. The relations for $s=2$ and $s=4$ have regions of negative slope, the relation for $s=1$ has a region of zero slope, and that for $s=0.5$ is everywhere positive. $D$, The same relation as in $C$ above for $s=2$, plotted as a V-I relation to correspond to the text figures. The arrows and dashed lines indicate the hysteresis in voltage that would occur with rising and falling current ramps. $I_{1}$ and $I_{2}$ are rheobasic currents for transitions from high to low conductance and low to high conductance, respectively. $U_{1}$ is the rheobasic voltage threshold for the transition from the high to low conductance condition, and $U_{2}$ represents the voltage achieved after the transition; $U_{3}$ and $U_{4}$ are corresponding voltages at rheobase for transition from the low to high conductance state. 
A

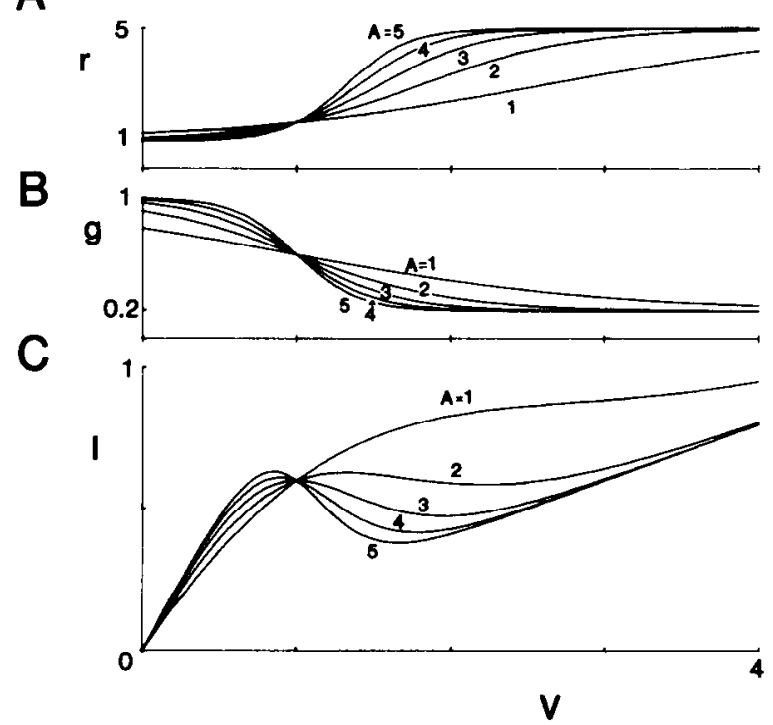

D

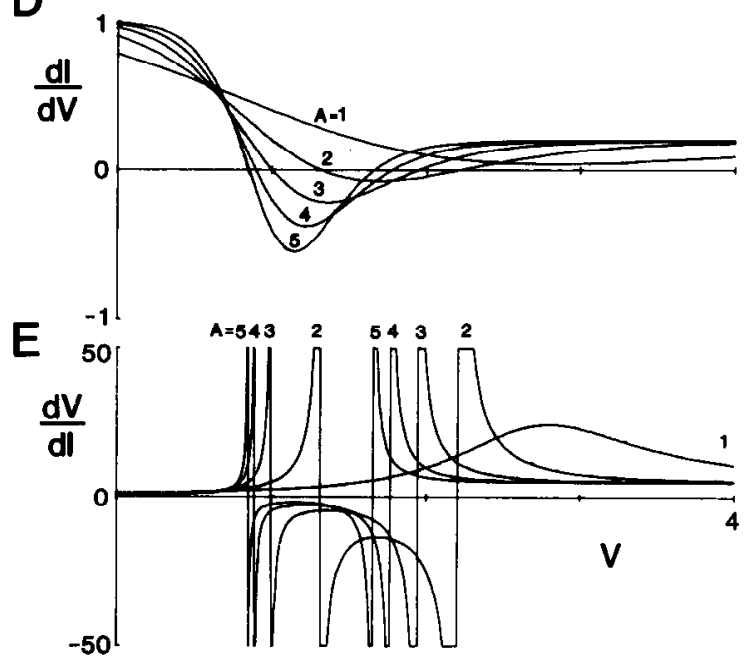

Figure A2. Fffects of a gradually changing resistance or conductance on I-V relations, slope conductances, and slope resistances. $A$ and $B$, Plots of the relation between resistance and voltage and between conductance and voltage according to text equation 6 . The parameter representing voltage sensitivity, $A$, is given values between 1 and 5 and $g_{\max } / g_{\min }=0.2$. Voltage is plotted in units of $V_{0}$ in this and subsequent graphs. Resistance and conductance are plotted in units of $r_{\min }$ and $g_{\max }$, respectively. $C$, The corresponding I-V relations. For $A \geq 2$ there is a negative slope region which becomes steeper as $A$ increases. Current is plotted in units of $g_{\max } V_{0}$ in this and subsequent graphs. $D$, The corresponding relations of slope conductance $d I / d V$ and voltage. For greater $A$, the slope conductance decreases more rapidly and becomes negative at smaller voltages. $E$, The corresponding relations of slope resistance $d V / d I$ and voltage. The discontinuities at transitions between positive and negative values are indicated by vertical lines.

particular voltage range and have what is termed an Sshaped characteristic (cf. Cole, 1968). For the voltagedependent conductance whose I-V relation has a region of negative slope, the conductance vs. current relation is three-valued over the current range where the slope is negative. Thus, this form of V-I relation implies an element that one characterizes as voltage rather than current dependent. Similarly, for a current-dependent conductance, the conductance vs. voltage relation is three-valued in voltage for voltages corresponding to the region of negative slope in the $I-V$ relation. If there is no negative slope region in the I-V relation, conductance is single valued for both voltage and current, and characterization as voltage or current dependent must be based on other considerations.

The linear increases in resistance of Figure $\mathrm{A} 1 A$, although convenient in illustrating the general requirements for a negative slope in the I-V relation, are unrealistic in having abrupt changes in the slope of the $r-V$ relation. Further information is obtained where changes in voltage are more gradual, as in actual conductances. A useful example is the Boltzmann relation which with appropriate parameters describes the voltage dependence of gap junctional conductance in amphibian blastomeres (text equation 6: $g=1 / r=\left(g_{\max }-g_{\min }\right) /\{1+$ $\left.\left.\exp \left[A\left(V-V_{0}\right)\right]\right\}+g_{\text {min }}\right)$. Figure A2, $A$ and $B$, shows $r$ - $V$ and $g-V$ plots for different values of $A$. Voltage is plotted as multiples of $V_{0}$, the voltage at which the conductance change is half-maximal. Conductance is plotted in units of $g_{\max }$ and resistance in units of $r_{\min } . A$ is a constant affecting the slope of the decrease in conductance with voltage, and variation in $A$ is analogous to variation in $s$ in the previous calculations. The value of $A=3.2$ corresponds approximately to the voltage sensitivity of blastomere junctional conductance for which $V_{0}=14.7 \mathrm{mV}$. To show both high and low conductance limbs of the VI relations on the same graph, $g_{\min }$ was taken as $0.2 g_{\max }$ (rather than $0.04 g_{\max }$ as for the actual junctional conductance).

The corresponding I-V relations are shown in Figure A2C and the slope conductance $d I / d V$ as a function of $V$ is shown in Figure A2D. For $A=1$ the slope conductance is everywhere positive (similar to $s=0.5$ in Fig. $\mathrm{A} 1 C$ ). For $A=2$ there is a region of slightly negative slope which becomes steeper for larger $A$ (similar to $s>1$ in Fig. A1C). (When $A$ is increased without limit, the negative slope region approaches the vertical at $V_{0}$.) The slope conductance is well behaved in the sense that in the transition region between high and low conductance, the slope conductance moves toward more negative values as $A$ is increased but remains a continuous function of voltage. Since the slope resistance $d V / d I$ is the inverse of the conductance, where the slope conductance goes through zero, slope resistance is a discontinuous function of voltage (Fig. A2E). As an I-V relation levels off and enters a negative slope region, the slope resistance passes through plus and minus infinity to reach finite negative values.

For a given value of $A$, changing $V_{0}$ shifts the $r-V$ and g- $V$ relations along the voltage axis. Increasing $V_{0}$ shifts the relations to the right and makes the right side of inequality (2) smaller than for corresponding points on the unshifted curve. Thus the slope conductance of the I-V relation becomes less positive or more negative in the 
transition region. Decreasing $V_{0}$ shifts the relations to the left and makes the right side of inequality (2) larger than for corresponding points on the unshifted curve. (For $V_{0} \leq 0$ there is no region of negative slope for any value of $A$.) Similarly, for a given value of $A$, the steepness of the transitions in the $r-V$ and $g-V$ plots is increased by increasing $r_{\max }$ or decreasing $g_{\min }$, making the slope conductance of the $\mathrm{V}$-I relation less positive or more negative in the transition region.

Bistability of cell coupling. To model coupled cells, additional conductances corresponding to nonjunctional membranes are introduced ( $g_{1}$ and $g_{2}$ in text Fig. 1). Consider a fixed postjunctional conductance $g_{2}$ in series with a voltage-dependent junctional conductance $g_{j}$ with parameters set at $A=4$ and $g_{\min }=0.2 g_{\max }$ as in Figure A2. Initially, the parallel conductance $g_{1}$ of cell 1 is taken as zero. At any current the voltage across the two conductances, corresponding to $V_{1}$, is the sum of the voltages $V_{j}$ and $V_{2}$ across $g_{j}$ and $g_{2}$, respectively (Fig. A3A).

The V-I relations in Figure $A 3 B$ are altered in shape by changing the ratio $m=g_{\max } / g_{2}$. For $m \cong 0, g_{2}$ is large compared to $g_{\max }$ and the V-I relation is unchanged. For larger values of $m$ (smaller $g_{2}$ ), the current range over which bistability is observed remains the same, as do the magnitudes of the voltage steps in transitions from high to low conductance states. However, the fractional changes in voltages during the transitions (corresponding to $U_{1} / U_{2}$ and $U_{4} / U_{3}$ in Fig. $\mathrm{A} 1 D$ ) are reduced. Because of the gradual changes in conductance with voltage, these ratios are somewhat less than $g_{\min } / g_{\max }$.

As the value of $m$ increases, an important change occurs. The slope of the transition from high to low conductance limbs of the V-I relation becomes flatter and then positive (between $m=2$ and $m=4$ for the conductance parameters illustrated). The V-I relation now exhibits bistability in current under constant voltage conditions as well as in voltage under constant current conditions. It is easily seen graphically (Fig. A3A) from addition of voltages across $g_{2}$ and $g_{j}$ that the transition region of the overall V-I relation will be horizontal (zero conductance) when the slope of the $V_{2}-I$ line is equal and opposite to the negative slope in the transition region. If $g_{\text {neg }}$ is the most negative slope conductance of $g_{j}$, the conductance of $g_{j}$ and $g_{2}$ in series at the corresponding current is $g_{\text {neg }} g_{2} /\left(g_{\text {neg }}+g_{2}\right)$. The criterion for bistability in current is that this conductance be positive or that $g_{2}$ $<-g_{\text {neg }}$.

The dashed lines and arrows in Figure $\mathrm{A} 3 B$ for the plot where $m=6$ indicate the hysteresis in current that would be exhibited during a gradually rising and falling ramp of voltage. (The hysteresis in voltage when current is varied remains of similar form as without the series conductance $g_{2}$ because the same current still flows through $g_{j}$.) The bistability of current for a given voltage is analogous to the problem encountered in voltage clamping an excitable patch of membrane when there is a significant series resistance (Cole, 1968).

If there is a nonzero conductance $g_{1}$ in parallel with $g_{i}$ and $g_{2}$ in series, the current at a given voltage is the sum of the currents through $g_{1}$ and through $g_{j}$ and $g_{2}$. By inspection of Figure $\mathrm{A} 4 A$, one sees that for a simple negative slope relation of $\boldsymbol{g}_{j}$ and $\boldsymbol{g}_{2}$, the region of bistability in current narrows and disappears as $g_{1}$ is increased. The value of $g_{1}$ at which the negative slope region disappears is equal to the magnitude of the maximum negative slope conductance for $g_{j}$ in series with $g_{2}$ which is given by $g_{n e g} g_{2} /\left(g_{\text {neg }}+g_{2}\right)$ (see the curve for $1 / k=g_{1} /$ $\left.g_{2}=0.5\right)$. Thus, even if the relation between $V_{j}+V_{2}$ and junctional current has a negative slope region like those illustrated, a large enough $g_{1}$ can prevent bistability. (Shunting by $g_{1}$ has the same effect on the V-I relations as increasing $g_{\min }$ in the relation between $\boldsymbol{g}_{j}$ and voltage.)

If a conductance $g_{1}$ shunts a V-I relation like that on the left in Figure A4B, the bistabilities in voltage and in

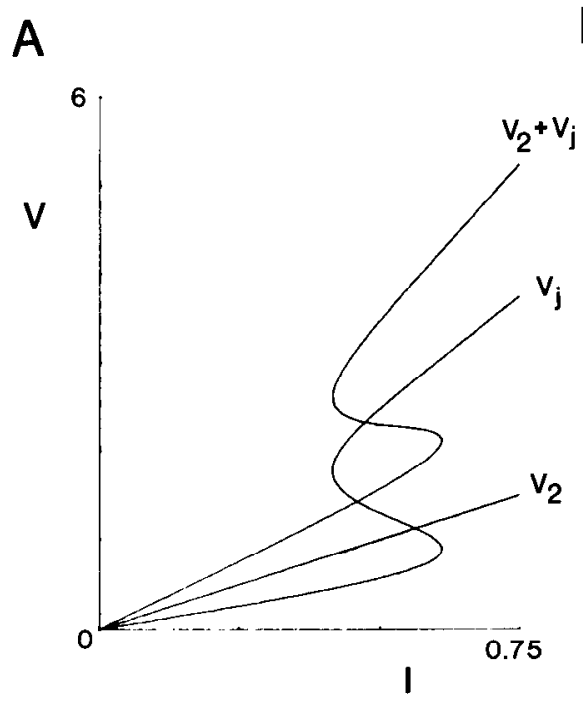

B

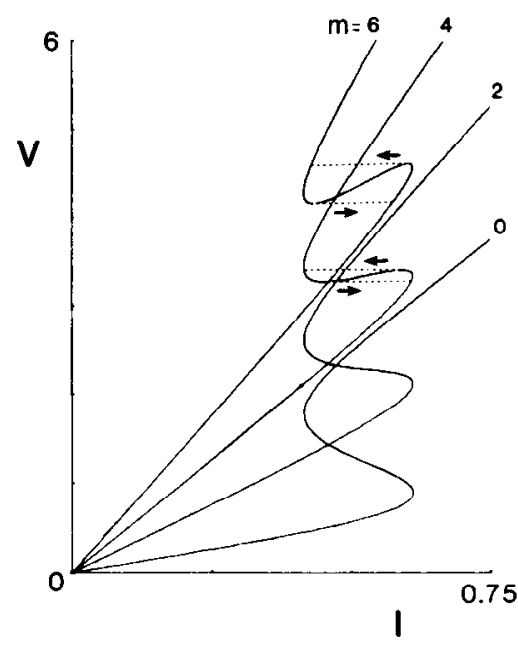

Figure A3. V-I relations of a postjunctional conductance $g_{2}$ in series with a junctional conductance whose V-I relation has a negative slope (the curve for $A=4$ in Figure $\mathrm{A} 2 C$ ). $A$, The voltage across $g_{j}$ and $g_{2}$ in series is the sum of the voltages across each ( $V_{j}$ and $V_{2}$, respectively). $B$, Effects of decreasing $g_{2}$ while holding $g_{\max }$ constant. Curves are labeled with the parameter $m=g_{\max }$ ) $g_{2}$. For large enough values of $m$, the V-I relation becomes bistable in voltage as well as current. The arrows and dashed lines for the curves where $m=4$ and $m=6$ indicate the hysteresis in current with ramp changes in voltage. 
A
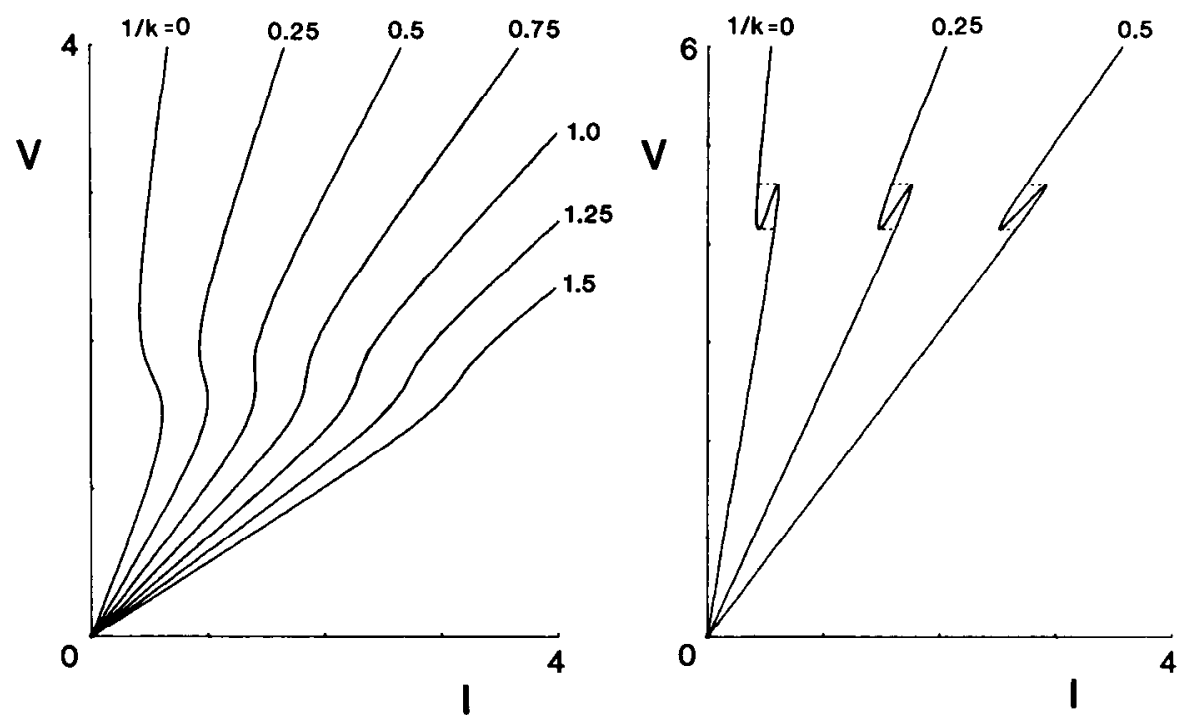

Figure A4. Effect of prejunctional conductance $g_{1}$ on V-I relations of junctional and postjunctional conductances, $g_{j}$ and $g_{2}$, in series. For a given voltage, total current is the sum of that through $g_{1}$ and that through $g_{j}$ and $g_{2}$. A, V-I relations for increasing $g_{1}$ shunting a V-I relation of a $g_{j}, g_{2}$ combination with a region of negative slope (curves like those in Fig. A3B for $n=2$ ). Values of $g_{1}=g_{2}(1 / k)$ are given for $1 / k$ equal to 0 to 1.5 in steps of 0.25 . The region of negative slope becomes more vertical and for $1 / k \geq 0.75$ the slope is everywhere positive. $B, \mathrm{~V}$-I relations for $g_{1}$ shunting a V-I relation of a $g_{j}$, $g_{2}$ combination with a region of bistability in voltage (curve for $m=6$ in Fig. A3B). Increasing $g_{1}$ does not prevent bistability in either current or voltage.

current remain no matter how large $g_{1}$ becomes, as indicated by the V-I relation on the right. The voltage range over which bistability in current occurs remains constant, and the change in current through $g_{j}+g_{2}$ remains constant (although a smaller fraction of the total).

Voltage dependence and intercellular signaling. To relate the foregoing analysis to intercellular communication one must examine the relative values of the postjunctional potentials for high and low junctional conductance states. In general, increasing $V_{1}$ has the opposing effects on $V_{2}$ of decreasing the coupling coefficient while increasing the voltage $\left(V_{1}\right)$ by which it is multiplied. The efficacy of signaling is increased both by greater voltage sensitivity of the conductance so that the change in $V_{1}$ required to change $g_{j}$ is smaller, and by having $g_{\text {min }}$ small compared to $g_{\max }$ so that a greater reduction in coupling is possible. If $V_{1}$ is varied between voltages that change transjunctional voltage between $U_{1}$ and $U_{3}$ so as to maximize the change in junctional conductance (from $g_{\max }$ to $g_{\min }$, assuming abrupt changes in slope as in Fig. A1B) we obtain:

$$
\begin{gathered}
V_{2}=V_{1} \frac{g_{\max }}{g_{\max }+g_{2}} \\
V_{2}^{\prime}=V_{1}^{\prime} \frac{g_{\min }}{\boldsymbol{g}_{\min }+g_{2}}
\end{gathered}
$$

where $U_{1}=V_{1}-V_{2}$ and $U_{3}=V_{1}^{\prime}-V_{2}^{\prime}$ and primes denote values at low junctional conductance. If $n=g_{\max } /$ $g_{\min }$, the ratio of postjunctional voltages is simply:

$$
\frac{V_{2}}{V_{2}^{\prime}}=n \frac{U_{1}}{U_{3}}
$$

which is independent of $m$, the ratio between $g_{\max }$ and $g_{2}$. Large $m$ (small $g_{2}$ ) will increase the postjunctional potential but also will increase the prejunctional potential required to change $g_{j}$ between $g_{\max }$ and $g_{\min }$.

The relevance of bistability to electrical control of junctional conductance is that brief electrical changes can cause switching between stable states of coupling and only small perturbations are required near thresholds. Optimization of signaling depends on the mode of prejunctional control. At the one extreme, changes in cell 1 could be equivalent to current clamp of $g_{j}$ and $g_{2}$ in series, if $g_{1}$ were small and current was generated by a voltage-insensitive electrogenic pump. At the other extreme, changes in cell 1 could be equivalent to voltage clamp of $g_{1}$ and $g_{2}$ in series if cell 1 were of high conductance. For given $g_{j}$ and $g_{2}$, increasing $g_{1}$ increases the changes in $V_{2}$ during transitions because $V_{1}$ changes less. However, the tendency toward bistability can be reduced, as noted above. Furthermore, lowering $g_{1}$ may increase the metabolic cost of shifting $V_{1}$.

The voltage range within which bistability in current occurs widens as $m$ increases. The limiting value of the fraction of the threshold voltage equals the fraction of threshold current over which bistability occurs $\left[\left(I_{1}-\right.\right.$ $\left.I_{2}\right) / I_{1}$ in Fig. A3]. However, large values of $m$ are required to approach this value and the changes in $V_{2}$ associated with changes in $g_{j}$ become small.

Where there is bistability of voltage at constant current, the junctional conductance will vary almost completely between $g_{\max }$ and $g_{\min }$ (transitions from steady state values near thresholds may be smaller). The associated postjunctional voltages in coupled and uncoupled states $\left(V_{2}\right.$ and $V_{2}^{\prime}$ above) are described by (from text 
equation 2):

$$
\begin{gathered}
V_{2}=\frac{g_{\text {max }}}{g_{1} g_{2}+g_{1} g_{\text {max }}+g_{2} g_{\text {max }}} I \\
V_{2}^{\prime}=\frac{g_{\text {min }}}{g_{1} g_{2}+g_{1} g_{\text {min }}+g_{2} g_{\text {min }}} I
\end{gathered}
$$

If $g_{2}=k g_{1}$ and $m$ and $n$ are defined as before, the ratio between the voltages is given by:

$$
\frac{V_{2}}{V_{2}^{\prime}}=\frac{(1+k) m+n}{(1+k) m+1}
$$

For large $k$ (small $g_{1}$ ) this ratio approaches 1 because constant current is applied to $g_{j}$ and $g_{2}$ in series and the voltage across $g_{2}$ is independent of the value of $g_{j}$. For large $m$ (large $g_{\max }$ ) this ratio also approaches 1 because the change in $g_{j}$ does not affect the current through $g_{2}$. At these extremes the postjunctional cell cannot detect the effects of changes in $g_{j}$. For small $m$ (small $g_{\max }$ ) the ratio approaches a maximum of $n$, but the postjunctional voltages become vanishingly small and of no signaling value.

If $k$ is very small (large $g_{1}$ ) the result is equivalent to voltage clamp, and the ratio approaches $(m+n) /(m+$ 1). ('This result differs from equation 3 because constancy of $V_{1}$ is assumed.) Increasing $k$ (decreasing $g_{1}$ ) decreases this ratio at any value of the other parameters because $V_{1}$ is increased when $g_{j}$ decreases, tending to oppose the effect of decreasing $g_{j}$ on $V_{2}$.

Because the maximum value of the ratio is obtained when $V_{2}$ and $V_{2}^{\prime}$ are vanishingly small, a better measure of efficacy of signaling is the difference in postjunctional voltages in coupled and uncoupled states normalized with respect to prejunctional voltage. Thus:

$$
\frac{V_{2}-V_{2}^{\prime}}{V_{1}}=\frac{\mathrm{m}(\mathrm{n}-1)}{(\mathrm{m}+1)[(1+\mathrm{k}) \mathrm{m}+\mathrm{n}]}
$$

This ratio equals zero for $m=0$ (small $g_{\max }$ ), for $m=\infty$ (large $g_{\max }$ ), and for $k=\infty$ (small $g_{1}$; constant current through $g_{j}$ and $g_{2}$ ). It is possible to show that as $m$ is varied the maximum value of this ratio occurs at $m=$ $\sqrt{n /(1+k)}$. For amphibian junctions $n \cong 20$. If $k=1$, that is, $g_{1}=g_{2}$, the maximum occurs at $m \cong 3$ and has a value of about 0.5. A family of curves of $\left(V_{2}-V_{2}^{\prime}\right) / V_{1}$ as a function of $m$ is shown in Figure A5A for $n=20$ and various values of $k$. The maxima occur in relatively flat regions of the curves and change slowly after $m$ exceeds 2 or 3 . For voltage dependence of amphibian junctions the magnitude of the most negative slope conductance is about $1 / 3 g_{\max }$ (Fig. A3A), and for $m$ larger than about 3 , bistability is present whatever the value of $g_{1}$ (Fig. A3B). For smaller $m$, bistability can be blocked if $g_{1}>-g_{2} g_{\text {neg }} /$ $\left(g_{2}+g_{\text {neg }}\right)$ from which $m>-\left(g_{\max } / g_{\text {neg }}\right) /(k+1)$ where $k=g_{2} / g_{1}$. These values of $m$ above which bistability will be obtained are indicated on each curve in Figure A5A. Near maximal changes in $g_{j}$ would require somewhat greater values of $m$. The calculations show that the amphibian system is indeed bistable for values of $g_{\max } / g_{2}$ where the calculated difference in postjunctional voltages are maximal.

A more widely applicable case than a pair of cells may be an infinite chain of identical cells. It is possible to show that where the input conductance into any junction

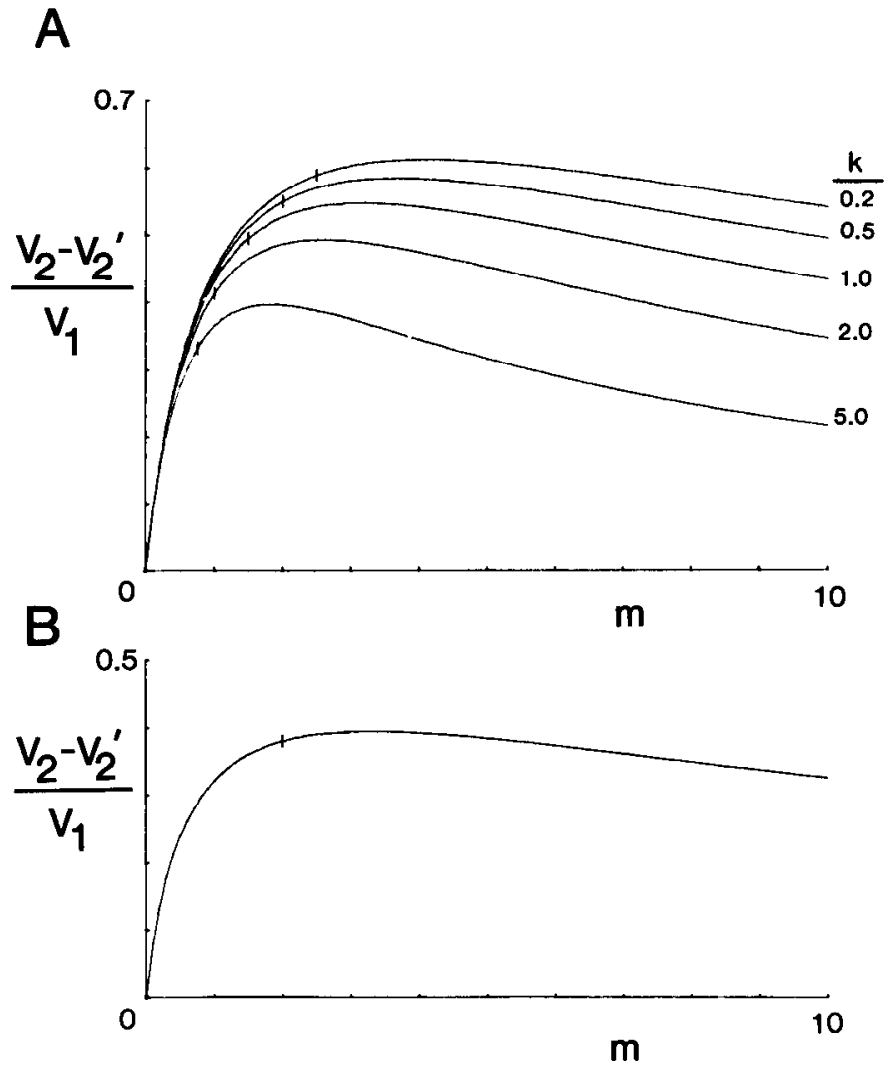

Figure A5. Efficacy of signaling measured as difference in postjunctional potentials in coupled and uncoupled states. $V_{2}$ - $V_{2}^{\prime}$ normalized with respect to prejunctional potential $V_{1}$ is plotted as a function of the ratio $m=g_{\max } / g_{2}$. $A$, For a pair of cells (equation 4), plots are shown as a function of $m=g_{\max } / g_{2}$ for $n=g_{\max } / g_{\min }=20$, the appropriate value for amphibian junctions, and for different values of $k=g_{2} / g_{1}$. The curves rise to relatively flat maxima. The vertical lines on each curve indicate the value which $m$ must exceed for bistability to occur. $B$, For an infinite chain of cells (equation 5), a single plot is shown for $n=20$ since $g_{1}=g_{2}$. The curve is even flatter than for a cell pair. Bistability is obtained for $m$ greater than 2 (vertical line).

is $g_{i n}$ and each nonjunctional conductance is $g_{2}$, the ratio $g_{\text {in }} / g_{2}$ is:

$$
g_{\text {in }}=-1 / 2+1 / 2 \sqrt{1+4 m}
$$

where, as before, $m=g_{\max } / g_{2}$. It can be assumed that in the uncoupled condition the conductance of the junctions of only the directly polarized cell will have become of low conductance because in the coupled condition transjunctional potential decreases exponentially with distance and the potentials will be largest across the nearest junctions (in the calculations shown in text Fig. 12, the cells were not identical). In this case it can be shown that the ratio of $V_{2}$ to $V_{2}^{\prime}$ is given by:

$$
V_{2} / V_{2}^{\prime}=\frac{(3+\sqrt{1+4 m}) m+(1+\sqrt{1+4 m}) n}{(3+\sqrt{1+4 m}) m+(1+\sqrt{1+4 m})}
$$

The limits of this ratio as the parameters are varied are the same as for a pair of cells, and the normalized difference in postjunctional potentials is again a better measure of signaling. The difference as a function of $m$ is 
given by:

$$
\begin{aligned}
\frac{V_{2}-V_{2}^{\prime}}{\mathrm{V}_{1}} & =\frac{m}{m+1 / 2+1 / 2 \sqrt{1+4 m}} \\
& -\frac{m \sqrt{1+4 m}}{(2+\sqrt{1+4 m}) m+(1 / 2+1 / 2 \sqrt{1+4 m}) n}
\end{aligned}
$$

shown in Figure $\mathrm{A} 5 B$ for $n=20$. The curve is very flat in the region of the maximum and changes very slowly once $m$ exceeds 2 . Given that $-g_{\max } / g_{\text {neg }}=3$, the cells would exhibit bistability for $m>2$ by the same criterion as applied to a cell pair (except that $g_{1}=g_{2}$ and the postjunctional conductance equals $\left.g_{2}+g_{\text {in }}\right)$.

In summary, for a pair of cells, bistability of voltage with respect to current is favored by steeper and more complete voltage dependence of junctional conductance. Although shunting by the conductance of the prejunctional cell decreases the tendency toward bistability, shunting is necessary for there to be a reduction in junctional current and postjunctional potential. Bistability also is favored by a sufficiently low conductance of the postjunctional cell and can develop with respect to current as a function of voltage. However, lowering the conductance of the postjunctional cell decreases the fractional changes in voltage or current in the transitions between states and reduces the efficacy of signaling as well as requiring a larger prejunctional voltage. A broad optimum range where the junctional conductance is at least several times greater than the post junctional conductance can be defined.

\section{References}

Auerbach, A. A., and M. V. L. Bennett (1969) A rectifying synapse in the central nervous system of a vertebrate. J. Gen. Physiol. 53: 211-237.

Bennett, M. V. L. (1966) Physiology of electrotonic junctions. Ann. N. Y. Acad. Sci. 137: 509-539.

Bennett, M. V. L., and D. A. Goodenough (1978) Gap junctions, electrotonic coupling, and intercellular communication. Neurosci. Res. Program Bull. 16: 373-486.

Bennett, M. V. L., B. Hille, and S. Obara (1970) Voltage threshold in excitable cells depends on stimulus form. $\mathrm{J}$. Neurophysiol. 33: 585-594.

Bennett, M. V. L., M. E. Spira, and D. C. Spray (1978) Permeability of gap junctions between embryonic cells of Fundulus: A reevaluation. Dev. Biol. 65: 114-125.

Bennett, M. V. L., A. L. Harris, and D. C. Spray (1980) Regenerative changes in coupling of Ambystoma blastomeres mediated by voltage dependent junctions. Soc. Neurosci. Abstr. 6: 249 .

Bennett, M. V. L., D. C. Spray, and A. L. Harris (1981a) Electrical coupling in development. Am. Zool. 21: 407-422.

Bennett, M. V. L., D. C. Spray, and A. L. Harris (1981b) Gap junctions and development. Trends Neurosci. 4: 159-163.

Bennett, M. V. L., A. L. Harris, D. C. Spray, R. D. Ginzberg, and E. A. Morales (1982) Interactive effects of cytoplasmic acidification and voltage on conductance of gap junctions between squid blastomeres. Biophys. J. 37: 317a.

Blackshaw, S. E., and A. E. Warner (1976) Alterations in resting membrane properties during neural plate stages of development of the nervous system. J. Physiol. (Lond.) 255: 231-247.

Cole, K. S. (1968) Membrane, Ions and Impulses. University of California Press, Berkeley.

Elias, P. M., and D. S. Friend (1976) Vitamin-A-induced mucous metaplasia. An in vitro system for modulating tight and gap junction differentiation. J. Cell Biol. 68: 173-188.

Flickinger, R. A. (1949) A study of the metabolism of amphibian neural crest cells during their migration and differentiation in vitro. J. Exp. Zool. 112: 465-484.

Furshpan, E. J., and D. D. Potter (1959) Transmission at the giant motor synapses of the crayfish. J. Physiol. (Lond.) 145: 289-325.

Furshpan, E. J., and D. D. Potter (1968) Low resistance junctions between cells in embryos and tissue culture. Curr. Top. Dev. Biol. 3: 95-127.

Gierer, A. (1977) Biological features and physical concepts of pattern formation exemplified by hydra. Curr. Top. Dev. Biol. 11: 16-59.

Goodman, C. S., and N. C. Spitzer (1979) Embryonic development of identified neurons: Differentiation from neuroblast to neurone. Nature 280: 208-214.

Harris, A. L. (1979) Voltage dependence of junctional conductance in early amphibian embryos. Doctoral dissertation, Stanford University, Stanford, CA.

Harris, A. L., D. C. Spray, and M. V. L. Bennett (1981) Kinetic properties of a voltage dependent junctional conductance. J. Gen. Physiol. 77: 95-117.

Harris, A. L., D. C. Spray, and M. V. L. Bennett (1982) Control of intercellular communication by voltage dependence of junctional conductance. Biophys. J. 37: 285a.

Harrison, R. G. (1969) Organization and Development of the Embryo. Yale University Press, New Haven.

Horn, R., J. Patlak, and C. F. Stevens (1981) Sodium channels need not open before they inactivate. Nature 291: 426-427.

Jaffe, L. F. (1981) The role of ionic currents in establishing devclopmental pattern. Philos. Trans. R. Soc. Lond. (Biol.) 295: 553-566

Lawrence, T. S., W. H. Beers, and N. B. Gilula (1978) Transmission of hormonal stimulation by cell-to-cell communication. Nature 272: 501-506.

Merk, F. B., and N. S. McNutt (1972) Nexus junctions between dividing and interphase granulosa cells of the rat. J. Cell Biol. 55: 511-515.

Messenger, E. A., and A. E. Warner (1979) The function of the sodium pump during differentiation of amphibian embryonic neurons. J. Physiol (Lond.) 292: 85-105.

Neher, E., and J. H. Steinbach (1978) Local anaesthetics transiently block currents through single acetylcholine-receptor channels. J. Physiol (Lond.) 277: 153-176.

Nicholls, J. G., and D. Purves (1970) Monosynaptic chemical and electrical connections between sensory and motor cells in the central nervous system of the leech. J. Physiol. (Lond.) 209: 647-688.

Obaid, A. L., and B. Rose (1981) Junctional membrane resistance of Chironomus cell pairs depends on (nonjunctional) membrane potential. Biophys. J. 33: 106a.

Pitts, J. D., and M. E. Finbow (1977) Junctional permeability and its consequences. In Intercellular Communication, W. C. DeMello, ed., pp. 61-86, Plenum Press, New York.

Poo, M-m., J. W. Lam, N. Orida, and A. W. Chao (1979) Electrophoresis and diffusion in the plane of the cell membrane. Biophys. J. 26: 1-21.

Raper, J., and C. S. Goodman (1982) Transient dye coupling between developing neurons reveals patterns of intercellular communication during embryogenesis. In Cellular Communication During Ocular Development, J. B. Sheffield and S. R. Hilfer, eds., pp. 85-96, Springer-Verlag, New York.

Rose, B. (1970) Junctional membrane permeability: Restoration by repolarizing current. Science 169: 607-609.

Rose, B., and W. R. Loewenstein (1975) Permeability of cell junction depends on local cytoplasmic calcium activity. $\mathrm{Na}$ ture 254: 250-252.

Slack, C., and A. E. Warner (1975) Properties of surface and 
junctional membranes of embryonic cells isolated from blastula stages of Xenopus laevis. J. Physiol. (Lond.) 248: 97-120.

Smith, T. G., and F. Baumann (1969) The functional organization within the ommatidium of the lateral eye of Limulus. Prog. Brain Res. 31: 313-349.

Smith, T. G., F. Baumann, F. and M. G. F. Fuortes (1965) Electrical connections between visual cells in the ommatidium of Limulus. Science 147: 1446-1448.

Socolar, S. J., and A. L. Politoff (1971) Uncoupling cell junctions in a glandular epithelium by depolarizing current. Science 172: 492-494.

Spira, M. E., D. C. Spray, and M. V. L. Bennett (1980) Synaptic organization of expansion motoneurons of Navanax inermis. Brain Res. 195: 241-269.

Spitzer, N. C. (1980) Electrical uncoupling of vertebrate spinal cord neurons during development. Soc. Neurosci. Abstr. 6: 287.

Spitzer, N. C. (1982) Voltage and stage dependent uncoupling of Rohon-Beard neurons during embryonic development of Xenopus tadpoles. J. Physiol. (Lond.), in press.

Spray, D. C., A. L. Harris, M. V. L. Bennett, and P. G. Model (1978) Voltage controlled resistance and permeability of gap junctions between embryonic cells. Soc. Neurosci. Abstr. 4: 238.

Spray, D. C., A. L. Harris, and M. V. L. Bennett (1979) Voltage dependence of junctional conductance in early amphibian embryos. Science 204: 432-434.

Spray, D. C., A. L. Harris, and M. V. L. Bennett (1981a) Equilibrium properties of a voltage dependent junctional conductance. J. Gen. Physiol. 77: 77-95.

Spray, D. C., A. L. Harris, and M. V. L. Bennett (1981b) Gap junctional conductance is a simple and sensitive function of intracellular pH. Science 211: 612-615.

Spray, D. C., A. L. Harris, and M. V. L. Bennett (1982a) Comparison of $\mathrm{pH}$ and $\mathrm{Ca}$ dependence of junctional conductance. In Intracellular pH: Its Measurement, Regulation, and Utilization in Cellular Functions, R. Nuccitelli and D. Deamer, eds., pp. 445-461, Alan R. Liss, New York.

Spray, D. C., J. H. Stern, A. L. Harris, and M. V. L. Bennett (1982b) Gap junctional conductance: Comparison of sensitivities to $\mathrm{H}$ and $\mathrm{Ca}$ ions. Proc. Natl. Acad. Sci. U. S. A. 79: 441-445.

Spray, D. C., A. L. Harris, and M. V. L. Bennett (1982c) Control of intercellular communication via gap junctions. In Cellular Communication During Ocular Development, J. B. Sheffield and S. R. Hilfer, eds., pp. 57-84, Springer-Verlag, New York.

White, R. L., D. C. Spray, A. Carvalho, and M. V. L. Bennett (1982) Voltage-dependent gap junctional conductance between fish embryonic cells. Soc. Neurosci. Abstr. 8: 944.

Wolpert, L. (1969) Positional information and the spatial pattern of cellular differentiation. J. Theor. Biol. 25: 1-47. 